\title{
One-loop superstring six-point amplitudes and anomalies in pure spinor superspace
}

\author{
Carlos R. Mafra ${ }^{a}$ and Oliver Schlotterer ${ }^{b}$ \\ ${ }^{a}$ School of Natural Sciences, Institute for Advanced Study, \\ Einstein Drive, Princeton, NJ 08540, U.S.A. \\ ${ }^{b}$ Max-Planck-Institut für Gravitationsphysik, Albert-Einstein-Institut, \\ Am Muehlenberg, 14476 Potsdam, Germany \\ E-mail: mafra@ias.edu, olivers@aei.mpg.de
}

ABSTRACT: We present the massless six-point one-loop amplitudes in the open and closed superstring using BRST cohomology arguments from the pure spinor formalism. The hexagon gauge anomaly is traced back to a class of kinematic factors in pure spinor superspace which were recently introduced as BRST pseudo-invariants. This complements previous work where BRST invariance arguments were used to derive the non-anomalous part of the amplitude. The associated worldsheet functions are non-singular and demonstrated to yield total derivatives on moduli space upon gauge variation. These cohomology considerations yield an efficient organizing principle for closed-string amplitudes that match expectations from S-duality in the low-energy limit.

Keywords: Anomalies in Field and String Theories, Superspaces, Superstrings and Heterotic Strings

ARXIV EPRINT: 1603.04790 


\section{Contents}

1 Introduction 1

2 Review: the hexagon anomaly and its cancellation $\quad 2$

2.1 The pure spinor description of the anomalous gauge variation 2

2.2 Multiparticle kinematic building blocks 4

2.3 BRST invariants 5

2.4 BRST pseudo-invariants 6

$\begin{array}{lll}2.5 & \text { Worldsheet functions } & 7\end{array}$

3 The complete six-point amplitude of the open string $\quad 8$

3.1 The non-anomalous part of the worldsheet correlator 8

$\begin{array}{ll}3.2 & \text { The anomalous part of the worldsheet correlator }\end{array}$

3.2.1 Motivating the BRST pseudo-invariant worldsheet correlator $\quad 10$

$\begin{array}{lll}3.3 & \text { The BRST and gauge transformations as boundary terms } & 12\end{array}$

4 The complete six-point amplitude of the closed string $\quad 13$

$\begin{array}{lll}4.1 & \text { The one-loop five point function for closed strings } & 14\end{array}$

$\begin{array}{ll}4.2 \text { The six-point closed-string correlator } & 15\end{array}$

$\begin{array}{lll}4.3 & \text { Low-energy limits and S-duality of type IIB } & 15\end{array}$

$\begin{array}{ll}\text { 4.4 The BRST variation as a boundary term } & 17\end{array}$

$\begin{array}{llr}5 & \text { Conclusion and outlook } & 18\end{array}$

$\begin{array}{ll}\text { A Permutation behavior of the open-string correlator } & 19\end{array}$

B Comparison with the RNS computation $\quad 20$

$\begin{array}{ll}\text { B.1 The parity-even part } & 21\end{array}$

$\begin{array}{ll}\text { B.2 The parity-odd part } & 22\end{array}$

C Gauge transformation versus BRST transformation $\quad \mathbf{2 4}$

C.1 Gauge variation of multiparticle superfields 24

$\begin{array}{lll}\text { C.2 Gauge variation of BRST (pseudo-)invariants } 26 & 26\end{array}$

\section{Introduction}

Over the past decade, several superstring [1-6] and field-theory scattering amplitudes [7-9] have been computed in manifestly supersymmetric form using the pure spinor formalism [10]. Computations in the minimal pure spinor formalism relied extensively on the BRST invariance of the amplitude prescription as a way to organize the intermediate steps 
and to simplify the answers. At tree level, this method led to a general solution in closed form for the $n$-point integrand for both the open superstring [4] as well as its field-theory limit [7]. At higher loops - apart from the four-point one- and two-loop amplitudes of $[1-3,11]$ - the superstring computations of $[5,6]$ so far were restricted to the lowenergy limit of the integrand. This limit only receives contributions from a subset of the zero-modes of the pure spinor b-ghost and leads to a simpler analysis of OPE singularities among external vertex operators.

In 2012 [12], the one-loop open superstring $n$-point integrand restricted to the above zero-mode contributions of the b-ghost was computed in closed form in terms of scalar BRST invariants denoted by $C_{i \mid A, B, C}$. These BRST invariants were later given a recursive construction in terms of ten-dimensional SYM superfields including a general expansion in terms of field-theory tree amplitudes [13]. Although the permutation-invariant integrands in [12] yield the desired low-energy behavior, they fail to reproduce the hexagon gauge anomaly on the boundary of moduli space.

The long-term goal of this project is to lift the restriction of b-ghost zero-modes from the one-loop analysis of [12] in order to obtain the complete and supersymmetric $n$-point one-loop amplitudes of the open superstring. In this paper we take the first step and write the complete six-point one-loop integrand for open and closed superstrings in pure spinor superspace. These results reproduce the pure spinor analysis of the gauge anomaly in [14] and match previous computations done with the RNS formalism. But unlike the RNS answer which is restricted to gluon amplitudes (see [15] for the parity-even and $[16,17]$ for the parity-odd part), the result of this paper is fully supersymmetric and naturally unifies the contributions from both the even and the odd spin structures. Moreover, the worldsheet integrals for both open and closed strings are cast into a basis. For closed strings, a new factorized representation of the five-point kinematics paves the way for an efficient organization of the six-point result.

Since the gauge anomaly probes non-standard contributions from the b-ghost beyond the zero-mode analysis of [12], the six-point one-loop result of this paper harbors important insights about a difficult corner of the pure spinor formalism which currently inhibits further progress in multiloop computations.

\section{Review: the hexagon anomaly and its cancellation}

\subsection{The pure spinor description of the anomalous gauge variation}

The gauge variation of the six-point open-superstring amplitude at one loop using the pure spinor formalism was computed in [14]. This subsection briefly reviews that derivation.

The non-minimal pure spinor prescription to compute a one-loop amplitude in the type-I superstring with a $\mathrm{SO}(N)$ gauge group is given by [18]

$$
\mathcal{A}_{n}=\sum_{\text {top }} G_{\text {top }} \int_{0}^{\infty} \mathrm{d} t \int_{\Delta_{\text {top }}} \mathrm{d} z_{2} \mathrm{~d} z_{3} \ldots \mathrm{d} z_{n}\left\langle\mathcal{N}(b, \mu) V_{1} \prod_{j=2}^{n} U_{j}\left(z_{j}\right)\right\rangle .
$$

The sum is over the three worldsheet topologies at one-loop with $G_{\text {top }}$ and $\Delta_{\text {top }}$ denoting their corresponding Chan-Paton factors and integration domains for $z_{j}$. Denoting the gen- 
erators of $\mathrm{SO}(N)$ in the fundamental representation by $t^{a_{i}}$, the Chan-Paton factors for the cylinder with all particles attached to one boundary and the Möbius strip are given by $G_{P}=$ $N \operatorname{tr}\left(t^{a_{1}} t^{a_{2}} t^{a_{3}} t^{a_{4}} t^{a_{5}} t^{a_{6}}\right)$ and $G_{N}=-\operatorname{tr}\left(t^{a_{1}} t^{a_{2}} t^{a_{3}} t^{a_{4}} t^{a_{5}} t^{a_{6}}\right)$. When particles are attached to both boundaries of the cylinder one has, for example, $G_{N P}=\operatorname{tr}\left(t^{a_{1}} t^{a_{2}}\right) \operatorname{tr}\left(t^{a_{3}} t^{a_{4}} t^{a_{5}} t^{a_{6}}\right)$. The integration domains will be elaborated in section 3.3.

Furthermore, $t$ is the one-loop Teichmüller parameter and $\mu$ the Beltrami differential, $b$ is the b-ghost (see [18] for the expression in the non-minimal formalism and $[1,19]$ for its schematic form in the minimal formalism), and $(b, \mu) \equiv \int \mathrm{d}^{2} w b(w) \mu$. The massless vertices are [10]

$$
V=\lambda^{\alpha} A_{\alpha}, \quad U=\partial \theta^{\alpha} A_{\alpha}+\Pi^{m} A_{m}+d_{\alpha} W^{\alpha}+\frac{1}{2} N^{m n} F_{m n}
$$

with pure spinor $\lambda^{\alpha}$ subject to $\left(\lambda \gamma^{m} \lambda\right)=0$, linearized superfields $\left[A_{\alpha}, A_{m}, W^{\alpha}, F_{m n}\right]$ of ten-dimensional SYM [20] and worldsheet fields $\left[\partial \theta^{\alpha}, \Pi^{m}, d_{\alpha}, N^{m n}\right]$ of conformal weight $h=1$ whose OPEs can be found in [10]. Finally, $\mathcal{N}$ regulates the integration over the non-compact space of pure spinors [18].

As in the original derivation of $[21,22]$, the gauge variation of the amplitude can be computed directly by replacing the vertex operators by their gauge variation

$$
\delta V_{1}=Q \Omega_{1}, \quad \delta U_{2}=\partial \Omega_{2},
$$

where $\Omega_{j}$ are scalar superfields, and the BRST charge is defined by

$$
Q \equiv \lambda^{\alpha} D_{\alpha}, \quad D_{\alpha} \equiv \frac{\partial}{\partial \theta^{\alpha}}+\frac{1}{2}\left(\gamma_{m} \theta\right)_{\alpha} k^{m} .
$$

Since the total derivatives $\partial \Omega_{2} \equiv \frac{\partial \Omega_{2}}{\partial z_{2}}$ from the integrated vertex operators are suppressed by the boundary contribution $z_{i} \rightarrow z_{j}$ of the integrand, the gauge variation of the six-point amplitude becomes

$$
\begin{aligned}
\delta \mathcal{A}_{6} & =\sum_{\text {top }} G_{\text {top }} \int_{0}^{\infty} \mathrm{d} t \int_{\Delta_{\text {top }}} \mathrm{d} z_{2} \ldots \mathrm{d} z_{6}\left\langle\mathcal{N}(b, \mu)\left(Q \Omega_{1}\right) \prod_{j=2}^{6} U_{j}\left(z_{j}\right)\right\rangle \\
& =-\sum_{\text {top }} G_{\text {top }} \int_{0}^{\infty} \mathrm{d} t \frac{\partial}{\partial t} \int_{\Delta_{\text {top }}} \mathrm{d} z_{2} \ldots \mathrm{d} z_{6}\left\langle\mathcal{N} \Omega_{1} \prod_{j=2}^{6} U_{j}\left(z_{j}\right)\right\rangle .
\end{aligned}
$$

To arrive at the second line the BRST charge was integrated by parts. The only nonvanishing contribution comes from the energy momentum tensor $\{Q, b\}=T$ and gives rise to a factor of $(T, \mu)$ which in turn leads to a total derivative $\frac{\partial}{\partial t}$ on moduli space [23].

The correlator in the second line of (2.5) can be easily evaluated by considering the saturation of fermionic zero-modes of the fermionic field $d_{\alpha}$. It is well known [18] that at one loop the regulator $\mathcal{N}$ provides eleven zero-modes of $d_{\alpha}$, so the vertices contribute the remaining five in order for the variation (2.5) to be non-vanishing, $\left(d W_{2}\right)\left(d W_{3}\right)\left(d W_{4}\right)\left(d W_{5}\right)$ $\left(d W_{6}\right)$. Integrating the pure spinor zero-modes has the effect of replacing $d_{\alpha_{1}} d_{\alpha_{2}} d_{\alpha_{3}} d_{\alpha_{4}} d_{\alpha_{5}} \rightarrow$ $\left(\lambda \gamma^{m}\right)_{\alpha_{1}}\left(\lambda \gamma^{n}\right)_{\alpha_{2}}\left(\lambda \gamma^{p}\right)_{\alpha_{3}}\left(\gamma_{m n p}\right)_{\alpha_{4} \alpha_{5}}[24]$, and (2.5) becomes

$$
\left.\delta \mathcal{A}_{6} \sim K \sum_{\text {top }} G_{\text {top }} \int_{\Delta_{\text {top }}} \mathrm{d} z_{2} \ldots \mathrm{d} z_{6}\left\langle\prod_{j=1}^{n} e^{i k_{j} \cdot x\left(z_{j}, \bar{z}_{j}\right)}\right\rangle\right|_{t \rightarrow 0} ^{t \rightarrow \infty}
$$


with the following kinematic factor for the hexagon gauge anomaly [14]

$$
K \equiv\left\langle\Omega_{1}\left(\lambda \gamma^{m} W_{2}\right)\left(\lambda \gamma^{n} W_{3}\right)\left(\lambda \gamma^{p} W_{4}\right)\left(W_{5} \gamma_{m n p} W_{6}\right)\right\rangle
$$

The standard correlator of plane waves $e^{i k_{j} \cdot x\left(z_{j}, \bar{z}_{j}\right)}$ is detailed in section 2.5. The component expansion of (2.7) can be computed using the zero-mode integration prescription [10]

$$
\left\langle\left(\lambda \gamma^{m} \theta\right)\left(\lambda \gamma^{n} \theta\right)\left(\lambda \gamma^{p} \theta\right)\left(\theta \gamma_{m n p} \theta\right)\right\rangle=2880
$$

and, when restricted to gluonic fields with polarization vectors $e_{i}$, is proportional to $\epsilon_{m_{1} n_{1} \ldots m_{5} n_{5}} k_{2}^{m_{1}} e_{2}^{n_{1}} \ldots k_{6}^{m_{5}} e_{6}^{n_{5}}$. In the next sections the result (2.6) will be re-derived from the gauge variation of an explicit expression for the six-point amplitude at one loop.

\subsection{Multiparticle kinematic building blocks}

The zero-mode structure of the six-point one-loop amplitude in the pure spinor formalism (2.1) allows for two OPEs among massless vertex operators. Such OPEs can be recursively addressed using non-local multiparticle superfields $\mathcal{K}_{P} \in\left\{\mathcal{A}_{\alpha}^{P}, \mathcal{A}_{P}^{m}, \mathcal{W}_{P}^{\alpha}, \mathcal{F}_{P}^{m n}\right\}$ of ten dimensional SYM [13]. They are referred to as Berends-Giele currents and defined by

$$
\mathcal{K}_{P} \equiv \frac{1}{s_{P}} \sum_{X Y=P} \mathcal{K}_{[X, Y]}
$$

where the multiparticle label $P=12 \ldots p$ encompasses $p$ external legs. The sum in $(2.9)$ instructs to deconcatenate $P$ into non-empty words $X=12 \ldots j$ and $Y=j+1 \ldots p$ with $j=1,2, \ldots, p-1$. The shorthand $\mathcal{K}_{[X, Y]}$ is used to represent all the four types of superfields simultaneously. More explicitly [25],

$$
\begin{aligned}
\mathcal{A}_{\alpha}^{[P, Q]} & \equiv-\frac{1}{2}\left[\mathcal{A}_{\alpha}^{P}\left(k^{P} \cdot \mathcal{A}^{Q}\right)+\mathcal{A}_{m}^{P}\left(\gamma^{m} \mathcal{W}^{Q}\right)_{\alpha}-(P \leftrightarrow Q)\right] \\
\mathcal{A}_{m}^{[P, Q]} & \equiv-\frac{1}{2}\left[\mathcal{A}_{m}^{P}\left(k^{P} \cdot \mathcal{A}^{Q}\right)+\mathcal{A}_{n}^{P} \mathcal{F}_{m n}^{Q}-\left(\mathcal{W}^{P} \gamma_{m} \mathcal{W}^{Q}\right)-(P \leftrightarrow Q)\right] \\
\mathcal{W}_{[P, Q]}^{\alpha} & \equiv \frac{1}{2}\left(k_{P}^{m}+k_{Q}^{m}\right) \gamma_{m}^{\alpha \beta}\left[\mathcal{A}_{P}^{n}\left(\gamma_{n} \mathcal{W}_{Q}\right)_{\beta}-(P \leftrightarrow Q)\right] \\
\mathcal{F}_{P}^{m n} & \equiv k_{P}^{m} \mathcal{A}_{P}^{n}-k_{P}^{n} \mathcal{A}_{P}^{m}-\sum_{X Y=P}\left(\mathcal{A}_{X}^{m} \mathcal{A}_{Y}^{n}-\mathcal{A}_{X}^{n} \mathcal{A}_{Y}^{m}\right) .
\end{aligned}
$$

Multiparticle momenta for $P=12 \ldots p$ and their associated Mandelstam invariants are given by

$$
k_{P}^{m} \equiv k_{1}^{m}+k_{2}^{m}+\cdots+k_{p}^{m}, \quad s_{P} \equiv \frac{1}{2} k_{P}^{2} .
$$

Furthermore, we define the multiparticle version of the vertex operator $V$ in (2.2) as

$$
M_{P} \equiv \lambda^{\alpha} \mathcal{A}_{\alpha}^{P},
$$

such that $M_{i}=V_{i}$. The zero-mode saturation in the pure spinor one-loop amplitude prescription selects certain superfields from the integrated vertex operators $U$ in (2.2), such 
as $V_{1}\left(\lambda \gamma_{m} W_{2}\right)\left(\lambda \gamma_{n} W_{3}\right) F_{4}^{m n}$ in the four-point amplitude [1]. Promoting the superfields to their Berends-Giele currents such as $W_{i}^{\alpha} \rightarrow \mathcal{W}_{A}^{\alpha}$ suggests the following definitions [13, 26],

$$
\begin{aligned}
M_{A, B, C} & \equiv \frac{1}{3}\left(\lambda \gamma_{m} \mathcal{W}_{A}\right)\left(\lambda \gamma_{n} \mathcal{W}_{B}\right) \mathcal{F}_{C}^{m n}+(A \leftrightarrow B, C) \\
\mathcal{W}_{A, B, C, D}^{m} & \equiv \frac{1}{12}\left[\left(\mathcal{W}_{A} \gamma^{m n p} \mathcal{W}_{B}\right)\left(\lambda \gamma_{n} \mathcal{W}_{C}\right)\left(\lambda \gamma_{p} \mathcal{W}_{D}\right)+(A, B \mid A, B, C, D)\right] \\
M_{A, B, C, D}^{m} & \equiv \mathcal{W}_{A, B, C, D}^{m}+\left[\mathcal{A}_{A}^{m} M_{B, C, D}+(A \leftrightarrow B, C, D)\right] \\
M_{A, B, C, D, E}^{m n} & \equiv \mathcal{A}_{A}^{m} \mathcal{W}_{B, C, D, E}^{n}+\mathcal{A}_{A}^{n} M_{B, C, D, E}^{m}+(A \leftrightarrow B, C, D, E),
\end{aligned}
$$

which automatically capture the results of iterated OPEs. In (2.17) and later places, the notation $\left(a_{1}, \ldots, a_{p} \mid a_{1}, \ldots, a_{n}\right)$ instructs to sum over all possible ways to choose $p$ elements $a_{1}, a_{2}, \ldots, a_{p}$ out of the set $\left\{a_{1}, \ldots, a_{n}\right\}$, for a total of $\left(\begin{array}{l}n \\ p\end{array}\right)$ terms.

\subsection{BRST invariants}

The zero-mode bracket in (2.8) which picks up the unique scalar of order $\lambda^{3} \theta^{5}$ from the enclosed superfields converts BRST invariants $S(\lambda, \theta)$ into supersymmetric and gaugeinvariant components $\langle S(\lambda, \theta)\rangle[10]$. Moreover, BRST-exact superfields are annihilated, $\langle Q(E(\lambda, \theta))\rangle=0[10]$. These properties already motivate to study the BRST cohomology to foresee kinematic factors in field-theory and string amplitudes in pure spinor superspace. From the covariant BRST transformations of one-loop building blocks in (2.16) to (2.18),

$$
\begin{aligned}
Q M_{A} & =\sum_{X Y=A} M_{X} M_{Y} \\
Q M_{A, B, C} & =\sum_{X Y=A}\left(M_{X} M_{Y, B, C}-M_{Y} M_{X, B, C}\right)+(A \leftrightarrow B, C), \\
Q M_{A, B, C, D}^{m} & =\sum_{X Y=A}\left(M_{X} M_{Y, B, C, D}^{m}-M_{Y} M_{X, B, C, D}^{m}\right)+k_{A}^{m} M_{A} M_{B, C, D}+(A \leftrightarrow B, C, D),
\end{aligned}
$$

one can recursively construct BRST-invariant scalars [13] such as

$$
\begin{aligned}
C_{1 \mid 23,4,5} \equiv & M_{1} M_{23,4,5}+M_{12} M_{3,4,5}-M_{13} M_{2,4,5} \\
C_{1 \mid 234,5,6} \equiv & M_{1} M_{234,5,6}+M_{12} M_{34,5,6}+M_{123} M_{4,5,6}-M_{124} M_{3,5,6} \\
& -M_{14} M_{23,5,6}-M_{142} M_{3,5,6}+M_{143} M_{2,5,6}, \\
C_{1 \mid 23,45,6} \equiv & M_{1} M_{23,45,6}+M_{12} M_{45,3,6}-M_{13} M_{45,2,6}+M_{14} M_{23,5,6}-M_{15} M_{23,4,6} \\
& +\left[M_{124} M_{3,5,6}-M_{134} M_{2,5,6}+M_{142} M_{3,5,6}-M_{143} M_{2,5,6}-(4 \leftrightarrow 5)\right],
\end{aligned}
$$

and vectors [13] such as

$$
\begin{aligned}
C_{1 \mid 2,3,4,5}^{m} \equiv & M_{1} M_{2,3,4,5}^{m}+\left[k_{2}^{m} M_{12} M_{3,4,5}+(2 \leftrightarrow 3,4,5)\right], \\
C_{1 \mid 23,4,5,6}^{m} \equiv & M_{1} M_{23,4,5,6}^{m}+M_{12} M_{3,4,5,6}^{m}-M_{13} M_{2,4,5,6}^{m} \\
& +\left[k_{3}^{m} M_{123} M_{4,5,6}+(3 \leftrightarrow 4,5,6)\right]-\left[k_{2}^{m} M_{132} M_{4,5,6}+(2 \leftrightarrow 4,5,6)\right] \\
& +\left[k_{4}^{m} M_{14} M_{23,5,6}+k_{4}^{m} M_{142} M_{3,5,6}-k_{4}^{m} M_{143} M_{2,5,6}+(4 \leftrightarrow 5,6)\right] .
\end{aligned}
$$


Their gauge-invariant bosonic components $\left\langle C_{1 \mid A, B, C}\right\rangle$ and $\left\langle C_{1 \mid A, B, C, D}^{m}\right\rangle$ determined from the zero-mode prescription (2.8) can be downloaded from [27]. As detailed in section 3.1, the scalars in (2.21) enter one-loop open-string amplitudes [12] but fail to explain the hexagon anomaly in view of their BRST invariance $Q C_{1 \mid A, B, C}=0$. The vectors $C_{1 \mid A, B, C, D}^{m}$ in turn are essential to efficiently represent the interactions between left- and right-movers in closed-string amplitudes, see section 4.

\subsection{BRST pseudo-invariants}

The hexagon gauge anomaly can be equivalently seen from a breakdown of BRST invariance, see appendix $\mathrm{C}$ for further details. Hence, the superfields in the anomaly kinematic factor $(2.7)$

$$
\mathcal{Y}_{A, B, C, D, E} \equiv \frac{1}{2}\left(\lambda \gamma^{m} \mathcal{W}_{A}\right)\left(\lambda \gamma^{n} \mathcal{W}_{B}\right)\left(\lambda \gamma^{p} \mathcal{W}_{C}\right)\left(\mathcal{W}_{D} \gamma_{m n p} \mathcal{W}_{E}\right)
$$

are required to appear in the BRST variation of the six-point open-string amplitude. We will refer to gauge and BRST anomalies interchangeably in the rest of the paper.

The tensorial building block (2.19) selected by zero-mode arguments exhibits an anomalous BRST transformation of this type in its trace component [26],

$$
\begin{aligned}
Q M_{A, B, C, D, E}^{m n}= & {\left[\sum_{X Y=A}\left(M_{X} M_{Y, B, C, D, E}^{m n}-M_{Y} M_{X, B, C, D, E}^{m n}\right)\right.} \\
& \left.+2 k_{A}^{(m} M_{A} M_{B, C, D, E}^{n)}+(A \leftrightarrow B, C, D, E)\right]+\delta^{m n} \mathcal{Y}_{A, B, C, D, E} .
\end{aligned}
$$

The same anomaly building block $\mathcal{Y}_{A, B, C, D, E}$ appears in the context of a scalar anomaly current whose single-particle version reads [26]

$$
\begin{aligned}
\mathcal{J}_{2 \mid 3,4,5,6} & \equiv \frac{1}{2} A_{2}^{m}\left(M_{3,4,5,6}^{m}+\mathcal{W}_{3,4,5,6}^{m}\right), \\
Q \mathcal{J}_{2 \mid 3,4,5,6} & =k_{2}^{m} M_{2} M_{3,4,5,6}^{m}+\left[s_{23} M_{23} M_{4,5,6}+(3 \leftrightarrow 4,5,6)\right]+\mathcal{Y}_{2,3,4,5,6} .
\end{aligned}
$$

While the above definition suffices for the six-point amplitude, a general definition with multiparticle labels can be found in [26].

Instead of a BRST-invariant completion such as the scalars and vectors in (2.21) and (2.22), the recursions of [26] select the combinations

$$
\begin{aligned}
C_{1 \mid 2,3,4,5,6}^{m n} \equiv & M_{1} M_{2,3,4,5,6}^{m n}+2\left[k_{2}^{(m} M_{12} M_{3,4,5,6}^{n)}+(3 \leftrightarrow 4,5,6)\right] \\
& +2\left[k_{2}^{(m} k_{3}^{n)}\left(M_{123}+M_{132}\right) M_{4,5,6}+(2,3 \mid 2,3,4,5,6)\right] \\
P_{1|2| 3,4,5,6} \equiv & M_{1} \mathcal{J}_{2 \mid 3,4,5,6}+M_{12} k_{2}^{m} M_{3,4,5,6}^{m}+\left[s_{23} M_{123} M_{4,5,6}+(3 \leftrightarrow 4,5,6)\right]
\end{aligned}
$$

for the tensor (2.19) and the anomaly current in (2.25). Since their BRST variations are exclusively furnished by the anomaly superfields in (2.23),

$$
Q C_{1 \mid 2,3,4,5,6}^{m n}=-\delta^{m n} V_{1} \mathcal{Y}_{2,3,4,5,6}, \quad Q P_{1|2| 3,4,5,6}=-V_{1} \mathcal{Y}_{2,3,4,5,6}
$$

these superfields are referred to as BRST pseudo-invariants. The motivation for this terminology stems from the purely parity-odd bosonic components which appear in the corresponding gauge variations such as (2.7) [26]. This ties in with the linearized gauge transformations $e_{1} \rightarrow k_{1}$ of the expressions for $\left\langle P_{1|2| 3,4,5,6}\right\rangle$ and $\left\langle C_{1 \mid 2,3,4,5,6}^{m n}\right\rangle$ on the webpage [27]. 


\subsection{Worldsheet functions}

String amplitudes augment kinematic factors with worldsheet integrals where the former conspire to BRST invariants or pseudo-invariants once the integrals are reduced to a basis. At one loop, the worldsheet integrand comprises doubly-periodic functions of the insertion points $z_{i}$ of the vertex operators such as the bosonic Green function on a genus-one surface with modular parameter $\tau$,

$$
G_{i j} \equiv G\left(z_{i j} \mid \tau\right) \equiv \ln \left|\frac{\theta_{1}\left(z_{i j} \mid \tau\right)}{\theta_{1}^{\prime}(0 \mid \tau)}\right|^{2}-\frac{2 \pi}{\tau_{2}}\left(\operatorname{Im} z_{i j}\right)^{2},
$$

where $z_{i j} \equiv z_{i}-z_{j}$ and $\tau_{2} \equiv \operatorname{Im}(\tau)$. Derivatives w.r.t. the first argument of $\theta_{1}(z \mid \tau)$ are interchangeably denoted by a tick and by $\partial \equiv \frac{\partial}{\partial z}$. Exponentials of $(2.29)$ give rise to the Koba-Nielsen factor from the plane-wave correlator seen for instance in (2.6):

$$
\mathcal{I}\left(s_{i j}\right) \equiv\left\langle\prod_{j=1}^{n} e^{i k_{j} \cdot x\left(z_{j}, \bar{z}_{j}\right)}\right\rangle_{\tau}=\prod_{i<j}^{n}\left\{\begin{array}{lll}
\exp \left[\frac{1}{2} \alpha^{\prime} s_{i j} G_{i j}\right] & : & \text { closed string } \\
\exp \left[2 \alpha^{\prime} s_{i j} G_{i j}\right] & : & \text { open string }
\end{array},\right.
$$

see (2.14) for the conventions for Mandelstam invariants $s_{i j}$. As a main result of this paper, we give a representation for the six-point open-string integrand such that its BRST variation builds up the modular derivative of (2.30) required by the anomalous gauge variation (2.5). For this purpose, we recall a set of doubly-periodic functions $f^{(n)}\left(z_{i j} \mid \tau\right) \equiv$ $f_{i j}^{(n)}$ with $n=0,1,2, \ldots$ described in [28] which were identified as a convenient language for one-loop superstring amplitudes. In particular, it turns out that

$$
\begin{aligned}
& f_{i j}^{(1)} \equiv \partial_{i} G\left(z_{i j} \mid \tau\right)=\partial \ln \theta_{1}\left(z_{i j} \mid \tau\right)+2 \pi i \frac{\operatorname{Im}\left(z_{i j}\right)}{\tau_{2}} \\
& f_{i j}^{(2)} \equiv \frac{1}{2}\left\{\left(\partial \ln \theta_{1}\left(z_{i j} \mid \tau\right)+2 \pi i \frac{\operatorname{Im}\left(z_{i j}\right)}{\tau_{2}}\right)^{2}-\wp\left(z_{i j} \mid \tau\right)\right\}
\end{aligned}
$$

with symmetries $f_{i j}^{(1)}=-f_{j i}^{(1)}, f_{i j}^{(2)}=f_{j i}^{(2)}$ and Weierstraß function [29]

$$
\wp(z \mid \tau) \equiv-\partial^{2} \ln \theta_{1}(z \mid \tau)+\frac{\theta_{1}^{\prime \prime \prime}(0 \mid \tau)}{3 \theta_{1}^{\prime}(0 \mid \tau)}
$$

suffice to describe the six-point amplitude. They are related via Fay's identity as [28],

$$
f_{i j}^{(1)} f_{i k}^{(1)}+f_{j i}^{(1)} f_{j k}^{(1)}+f_{k i}^{(1)} f_{k j}^{(1)}=f_{i j}^{(2)}+f_{j k}^{(2)}+f_{k i}^{(2)},
$$

and one can show that the short-distance singularities of $\left(\partial \ln \theta_{1}\right)^{2}$ and $\wp$ drop out from (2.32), rendering $f_{i j}^{(2)}$ non-singular as $z_{i j} \rightarrow 0$. The relation of $f_{i j}^{(2)}$ with the $\tau$ derivative of the Green function (2.29) is explained and applied in section 3.3. The net result

$$
\frac{\partial}{\partial t^{\prime}} \mathcal{I}\left(s_{i j}\right) \sim \mathcal{I}\left(s_{i j}\right) \sum_{i<j}^{6} s_{i j} f_{i j}^{(2)}
$$

with $t^{\prime} \equiv 1 / t$ connects the derivative in moduli space appearing in the gauge variation (2.6) with the function $f_{i j}^{(2)}$ in the anomalous six-point correlator (3.7). 


\section{The complete six-point amplitude of the open string}

In applying the pure spinor one-loop prescription (2.1), the non-zero modes of the b-ghost lead to cumbersome CFT calculations. One way to address this difficulty is to use the BRST invariance of the pure spinor formalism as a guiding principle to write down the answers directly. This will be done in this section for the open-string six-point amplitude; the result contains two classes of kinematic factors: BRST invariants $\left(\mathcal{K}^{C}\right)$ and pseudoinvariants $\left(\mathcal{K}^{P}\right)$. Recalling the zero-mode prescription $\langle\ldots\rangle$ in $(2.8)$, our conventions are

$$
\mathcal{A}_{6}=\sum_{\text {top }} G_{\text {top }}\left\langle A_{6}^{\text {top }}\right\rangle, \quad A_{6}^{\text {top }} \equiv \int_{0}^{\infty} \frac{\mathrm{d} t}{t^{5}} \int_{\Delta_{\text {top }}} \mathrm{d} z_{2} \mathrm{~d} z_{3} \ldots \mathrm{d} z_{6} \mathcal{I}\left(s_{i j}\right)\left(\mathcal{K}_{6}^{C}+\mathcal{K}_{6}^{P}\right) .
$$

A separate analysis will be performed for each sector, and the pseudo-invariants $\mathcal{K}^{P}$ will shortly be defined such as to make contact with the kinematic factor (2.7) of the anomalous gauge variation.

\subsection{The non-anomalous part of the worldsheet correlator}

A gauge-invariant subsector of one-loop open-string amplitudes which describes the lowenergy behavior has been analyzed to all multiplicities in [12]. Its kinematic factors are captured by the BRST-closed scalars $C_{i \mid A, B, C}$ in pure spinor superspace as exemplified in (2.21). Their derivation considers only the zero-mode contributions from the b-ghost leading to the scalar building blocks (2.16) and follows from integration by parts identities of the worldsheet functions associated to OPE singularities to reduce the integrals to a basis. More specifically, products of worldsheet propagators (2.29) and $s_{i j}$ in (2.14),

$$
X_{i j} \equiv s_{i j} f_{i j}^{(1)}=s_{i j} \partial G_{i j},
$$

can be conveniently manipulated by discarding ${ }^{1}$ total derivatives acting on the KobaNielsen factor (2.30):

$$
\partial_{p} \mathcal{I}\left(s_{i j}\right)=\alpha^{\prime} \mathcal{I}\left(s_{i j}\right) \sum_{q \neq p} X_{p q}
$$

A basis of worldsheet functions in open- and closed-string correlators can be attained by removing any explicit appearance of the fixed insertion point $z_{1}$ along with $X_{1 j}$ through the addition of total derivatives (3.3) with respect to $z_{j}$.

In terms of the worldsheet functions (3.2) and the BRST invariants $C_{i \mid A, B, C}$, a permutation-invariant kinematic factor for the six-point amplitude (3.1) is given by [12]

$$
\begin{aligned}
\mathcal{K}_{6}^{C}= & {\left[X_{23}\left(X_{24}+X_{34}\right) C_{1 \mid 234,5,6}+X_{24}\left(X_{23}+X_{43}\right) C_{1 \mid 243,5,6}+(2,3,4 \mid 2,3,4,5,6)\right] } \\
& +\left[X_{23} X_{45} C_{1 \mid 23,45,6}+X_{24} X_{35} C_{1 \mid 24,35,6}+X_{25} X_{34} C_{1 \mid 25,34,6}+(6 \leftrightarrow 5,4,3,2)\right] .
\end{aligned}
$$

\footnotetext{
${ }^{1}$ Boundary terms in $z_{i}$ do not contribute since the exponential of $\alpha^{\prime} s_{i j} G_{i j}$ vanishes as $z_{i j}^{\alpha^{\prime}} s_{i j}$ for $z_{i} \rightarrow z_{j}$. This is obvious if $s_{i j}$ has a positive real part, whereas the vanishing for generic momenta follows from analytic continuation [30].
} 
As initially observed in [12], the scalar BRST invariants $C_{i \mid A, B, C}$ can be re-expressed in terms of color-ordered SYM tree amplitudes. At six points, the identities [13]

$$
\begin{aligned}
\left\langle C_{1 \mid 234,5,6}\right\rangle= & s_{56}\left[s_{45} A^{\mathrm{YM}}(1,2,3,4,5,6)-s_{35} A^{\mathrm{YM}}(1,2,4,3,5,6)\right. \\
& \left.-s_{35} A^{\mathrm{YM}}(1,4,2,3,5,6)+s_{25} A^{\mathrm{YM}}(1,4,3,2,5,6)\right] \\
\left\langle C_{1 \mid 23,45,6}\right\rangle= & s_{46} s_{36} A^{\mathrm{YM}}(1,2,3,6,4,5)-s_{56} s_{36} A^{\mathrm{YM}}(1,2,3,6,5,4) \\
& -s_{46} s_{26} A^{\mathrm{YM}}(1,3,2,6,4,5)+s_{56} s_{26} A^{\mathrm{YM}}(1,3,2,6,5,4),
\end{aligned}
$$

allow to straightforwardly express all the polarization dependence of (3.4) in terms of $A^{\mathrm{YM}}(\ldots)$. However, the above BRST-invariant integrand $\mathcal{K}_{6}^{C}$ cannot be the complete answer for the six-point open string amplitude since it would imply manifest gauge invariance. $^{2}$ In the following, we will show how the anomalous part of the amplitude can be described using the BRST pseudo-invariants derived in [26] and reviewed in section 2.4.

\subsection{The anomalous part of the worldsheet correlator}

In order to correctly describe the anomalous part of one-loop amplitudes, the kinematic factor $\mathcal{K}_{6}^{P}$ in (3.1) cannot be BRST invariant. According to (2.5), its BRST variation must add up to a total derivative in moduli space and reflect a parity-odd gauge variation. For this purpose, the notion of a pseudo BRST cohomology was introduced in [26] along with recursive method to construct pseudo-invariants of arbitrary multiplicity and tensor rank. Its scalar six-point representative $P_{1|2| 3,4,5,6}$ has been defined in (2.27), and its BRST variation $-V_{1} \mathcal{Y}_{2,3,4,5,6}$ in terms of the superfields (2.23) tie in with anomaly kinematic factor (2.7). That is why this superfield is suitable to describe the anomalous gauge variation of the six-point integrand.

Using the above pseudo-invariants, the anomalous part of the six-point correlator (3.1) will be argued to be

$$
\mathcal{K}_{6}^{P}=\left[s_{12} f_{12}^{(2)} P_{1|2| 3,4,5,6}+(2 \leftrightarrow 3,4,5,6)\right]+\left[s_{23} f_{23}^{(2)} P_{1|(23)| 4,5,6}+(2,3 \mid 2,3,4,5,6)\right],
$$

with $f_{i j}^{(2)}$ in $(2.32)$ and

$$
P_{1|(23)| 4,5,6} \equiv P_{2|3| 1,4,5,6}-P_{2|1| 3,4,5,6}+P_{1|2| 3,4,5,6} .
$$

Its BRST and gauge variations

$$
Q \mathcal{K}_{6}^{P}=-V_{1} \mathcal{Y}_{2,3,4,5,6} \sum_{i<j}^{6} s_{i j} f_{i j}^{(2)}, \quad \delta \mathcal{K}_{6}^{P}=-\Omega_{1} \mathcal{Y}_{2,3,4,5,6} \sum_{i<j}^{6} s_{i j} f_{i j}^{(2)}+Q(\ldots)
$$

will be identified as a boundary term in moduli space in section 3.3. Therefore the anomaly cancellation for gauge group $\mathrm{SO}(32)$ can be proven as in the RNS formalism and will not be repeated here $[16,17,21,22,31]$.

In contrast to the BRST-invariant kinematic factors $C_{i \mid A, B, C}$ in (3.5) and (3.6), the pseudo-invariant $P_{1|2| 3,4,5,6}$ cannot be expressed in terms of SYM tree-level subamplitudes. Two classes of tensor structures in its bosonic components [27] pose an obstruction:

\footnotetext{
${ }^{2}$ We are grateful to Michael Green for insisting on a clarification of this point.
} 
1. terms of the schematic form $\left(e_{i} \cdot k_{j}\right)^{6}$ where all the six gluon polarization vectors $e_{i}$ with $i=1,2, \ldots, 6$ are contracted with an external momentum

2. parity-odd terms involving the ten-dimensional Levi-Civita tensor $\epsilon_{m_{1} m_{2} \ldots m_{10}}$

It is easy to see from Feynman rules and worldsheet supersymmetry that parity-even contractions $\left(e_{i} \cdot k_{j}\right)^{6}$ are absent in tree amplitudes of both SYM and the open superstring. ${ }^{3}$

\subsubsection{Motivating the BRST pseudo-invariant worldsheet correlator}

The pseudo-invariants $P_{1|2| 3,4,5,6}$ in (2.27) are symmetric under permutations of $3,4,5,6$ whereas the "reference leg" 1 is singled out by the choice of unintegrated vertex $V_{1}$ in the amplitude prescription (2.1). This reasoning motivates to associate $P_{1|2| 3,4,5,6}$ with the worldsheet function $f_{12}^{(2)}$ in (2.32). Upon permutations in the integrated legs $2,3, \ldots, 6$, this assigns natural kinematic companions $P_{1|2| 3,4,5,6}, \ldots, P_{1|6| 2,3,4,5}$ to five instances $f_{12}^{(2)}, f_{13}^{(2)}, \ldots, f_{16}^{(2)}$ out of the 15 functions $\left\{f_{i j}^{(2)}, 1 \leq i<j \leq 6\right\}$.

The form of the remaining kinematic factors can be inferred from the symmetry properties of the anomalous correlator $\mathcal{K}_{6}^{P}$. In contrast to the permutation-invariant expression for $\mathcal{K}_{6}^{C}$ in (3.4), symmetry of $\mathcal{K}_{6}^{P}$ under exchange of the unintegrated leg $(1 \leftrightarrow 2)$ is slightly broken by the anomaly. This can be traced back to the different response of unintegrated and integrated vertex operator to gauge variations, see (2.3). The anomalous BRST variation (2.28) makes reference to $V_{1}$ in the prescription, and different choices of the unintegrated vertex are related by $[26]$

$$
Q \mathcal{Y}_{12,3,4,5,6}=V_{1} \mathcal{Y}_{2,3,4,5,6}-V_{2} \mathcal{Y}_{1,3,4,5,6}
$$

with a two-particle version $\mathcal{Y}_{12,3,4,5,6}$ of the anomaly building block (2.23). This BRST variation reproduces the antisymmetric part of the anomalous gauge variation (2.7), and a detailed account on the emergence of $\mathcal{Y}_{12,3,4,5,6}$ under antisymmetrization in $(1 \leftrightarrow 2)$ can be found in appendix A. Indeed, the kinematic coefficient of the function $f_{12}^{(2)}=f_{21}^{(2)}$ is symmetric up to the BRST generator in (3.10) [26],

$$
\left\langle P_{1|2| 3,4,5,6}\right\rangle=\left\langle P_{2|1| 3,4,5,6}-\mathcal{Y}_{12,3,4,5,6}\right\rangle
$$

We interpret the superfield $\mathcal{Y}_{12,3,4,5,6}$ as an anomaly-transporting term between external legs 1 and 2. Just as the anomalous gauge variation (2.7), its bosonic components are parity odd,

$$
\left\langle\mathcal{Y}_{12,3,4,5,6}\right\rangle=-\epsilon_{p_{3} p_{4} p_{5} p_{6} q_{1} q_{2} \ldots q_{6}} k_{3}^{p_{3}} k_{4}^{p_{4}} k_{5}^{p_{5}} k_{6}^{p_{6}} e_{1}^{q_{1}} e_{2}^{q_{2}} \cdots e_{6}^{q_{6}}
$$

see appendix B of [26] for a general argument.

Accordingly, the coefficient of $f_{23}^{(2)}$ cannot follow from a naive relabeling of the legs in the combination $f_{12}^{(2)} \leftrightarrow s_{12} P_{1|2| 3,4,5,6}$ since $Q P_{2|3| 1,4,5,6}=-V_{2} \mathcal{Y}_{1,3,4,5,6}$. However, we see

\footnotetext{
${ }^{3}$ For an exploitation of this property in the RNS formalism, see [32, 33].
} 
from (3.10) that the anomalous BRST variation can be corrected via $\mathcal{Y}_{12,3,4,5,6}$. In view of (3.11), the natural candidate to multiply the function $f_{23}^{(2)}$ is $P_{1|(23)| 4,5,6}$ in (3.8) with

$$
Q P_{1|(23)| 4,5,6}=-V_{1} \mathcal{Y}_{2,3,4,5,6}
$$

The $2 \leftrightarrow 3$ symmetry suggested by $f_{23}^{(2)}=f_{32}^{(2)}$ can be checked to hold,

$$
\begin{aligned}
\left\langle P_{1|(23)| 4,5,6}-P_{1|(32)| 4,5,6}\right\rangle & =\left\langle P_{1|2| 3,4,5,6}-P_{2|1| 3,4,5,6}+\operatorname{cyc}(1,2,3)\right\rangle \\
& =-\left\langle\mathcal{Y}_{12,3,4,5,6}+\mathcal{Y}_{23,1,4,5,6}+\mathcal{Y}_{31,2,4,5,6}\right\rangle=0,
\end{aligned}
$$

where the cyclic combination of $\mathcal{Y}$ 's in the second line is BRST trivial under six-particle momentum conservation $k_{123456}^{m}=0$ [26] (cf. (3.12) for the vanishing of the bosonic components). In the interpretation of $\left\langle\mathcal{Y}_{12,3,4,5,6}\right\rangle$ as an anomaly transportation term, the vanishing of (3.14) can be made plausible since the second line describes an anomaly transportation around a closed loop $1 \rightarrow 2 \rightarrow 3 \rightarrow 1$. Note that an alternative cohomology representation of $P_{1|(23)| 4,5,6}$ is given by [26]

$$
\begin{aligned}
\left\langle P_{1|(23)| 4,5,6}\right\rangle= & \frac{1}{2}\left\langle\left(k_{3}^{m}-k_{2}^{m}\right) C_{1 \mid 23,4,5,6}^{m}+P_{1|3| 2,4,5,6}+P_{1|2| 3,4,5,6}\right. \\
& \left.+\left[s_{34} C_{1 \mid 234,5,6}+s_{24} C_{1 \mid 324,5,6}+(4 \leftrightarrow 5,6)\right]\right\rangle .
\end{aligned}
$$

The symmetry properties of the anomalous correlator can be summarized as

$$
\left\langle\left.\mathcal{K}_{6}^{P}\right|_{1 \leftrightarrow 2}-\mathcal{K}_{6}^{P}\right\rangle=\left\langle\mathcal{Y}_{12,3,4,5,6}\right\rangle \sum_{i<j}^{6} s_{i j} f_{i j}^{(2)}, \quad\left\langle\left.\mathcal{K}_{6}^{P}\right|_{2 \leftrightarrow 3}-\mathcal{K}_{6}^{P}\right\rangle=0,
$$

see appendix A for a derivation from the amplitude prescription (2.1). The analysis in section 3.3 will also identify the failure of permutation invariance in $\left\langle\mathcal{K}_{6}^{P}\right\rangle$ as a boundary term.

In addition to the above plausibility arguments in superspace, we have explicitly tested the anomalous correlator (3.7) for consistency with the RNS computation of the six-gluon amplitude. The technical aspects of this consistency check are explained in appendix B. The RNS computation must be carried out separately for the parity-even and the parity-odd sector. The former is presented in B.1, mostly guided by the results of $[15,28,34,35]$. The parity-odd counterpart presented in appendix B.2 largely follows the computations in [16] apart from the presentation of worldsheet functions. In the pure spinor representation of the correlator in (3.7), both parity sectors are unified through the component expansion of the pseudo-invariants $\left\langle P_{1|2| 3,4,5,6}\right\rangle$ and $\left\langle P_{1|(23)| 4,5,6}\right\rangle$.

Moreover, we have checked that the field-theory limit of the above six-point amplitude reproduces the one-loop integrand of ten-dimensional SYM which has been derived in [8] from cohomology arguments. Upon dimensional reduction to $D=4$, the pseudoinvariant $P_{1|2| 3,4,5,6}$ and therefore the entire anomalous correlator (3.7) vanishes for MHV helicity configurations. Hence, the non-anomalous contribution (3.4) is sufficient to derive the BCJ representation of MHV amplitudes in [36] from the field-theory limit. 


\subsection{The BRST and gauge transformations as boundary terms}

In this subsection, we discuss the scalar integrals accompanying the anomalous BRST and gauge variations (3.9) of the six-point amplitude. In particular, they are now demonstrated to describe boundary terms in the moduli space of open-string worldsheets.

In order to relate the BRST variation $(3.9)$ of $\mathcal{K}_{6}^{P}$ to a total derivative with respect to the modular parameter, it is worthwhile to express the functions $f_{p q}^{(2)}$ in terms of the $\tau$ derivative of the bosonic Green function (2.29). For generic complex arguments, the heat equation $4 \pi i \frac{\partial \theta_{1}(z \mid \tau)}{\partial \tau}=\partial^{2} \theta_{1}(z \mid \tau)$ obeyed by the theta function in (2.33) implies that

$$
f_{p q}^{(2)} \equiv f^{(2)}\left(z_{p q} \mid \tau\right)=2 \pi i\left(\frac{\partial G_{p q}}{\partial \tau}+\frac{\operatorname{Im} z_{p q}}{\tau_{2}} \partial G_{p q}\right)+\frac{\theta_{1}^{\prime \prime \prime}(0 \mid \tau)}{3 \theta_{1}^{\prime}(0 \mid \tau)}-\frac{\pi}{\tau_{2}} .
$$

In a convenient parametrization of open-string worldsheets, the arguments $z_{p q}, \tau$ of the Green function (2.29) have constant real parts and are integrated over their imaginary parts $\nu_{p q} \equiv \nu_{p}-\nu_{q}$ and $t$ :

$$
\left(z_{p q}, \tau\right) \rightarrow \begin{cases}\left(i \nu_{p q}, i t\right) & : p \text { and } q \text { on the same cylinder boundary } \\ \left(i \nu_{p q}+\frac{1}{2}, i t\right) & : p \text { and } q \text { on different cylinder boundaries } \\ \left(i \nu_{p q}, i t+\frac{1}{2}\right) & : \text { Möbius strip }\end{cases}
$$

The integration domains $\Delta_{\text {top }}$ for vertex insertions in (2.1) and (3.1) are then given by

$$
\begin{aligned}
\Delta_{P} & =\left\{0 \leq \nu_{1} \leq \nu_{2} \leq \ldots \leq \nu_{6} \leq t\right\} \\
\Delta_{N} & =\left\{0 \leq \nu_{1} \leq \nu_{2} \leq \ldots \leq \nu_{6} \leq 2 t\right\} \\
\Delta_{N P} & =\left\{0 \leq \nu_{1}, \nu_{2} \leq t \text { and } 0 \leq \nu_{3} \ldots \leq \nu_{6} \leq t\right\}
\end{aligned}
$$

where $\Delta_{P}$ and $\Delta_{N}$ are adapted to the single-traces over $t^{a_{1}} t^{a_{2}} t^{a_{3}} t^{a_{4}} t^{a_{5}} t^{a_{6}}$, and $\Delta_{N P}$ refers to the non-planar cylinder diagram with color factor $G_{N P}=\operatorname{tr}\left(t^{a_{1}} t^{a_{2}}\right) \operatorname{tr}\left(t^{a_{3}} t^{a_{4}} t^{a_{5}} t^{a_{6}}\right)$. Hence, the functional dependence of $G_{p q}$ on the real parameters $\nu_{p q}$ and $t$ is given as follows in the three inequivalent configurations:

$$
G_{p q}=G\left(i \nu_{p q}+\delta \mid i t+\varepsilon\right), \quad(\delta, \varepsilon)= \begin{cases}(0,0) & : p \text { and } q \text { on the same cylinder boundary } \\ \left(\frac{1}{2}, 0\right) & : p \text { and } q \text { on different cylinder boundaries } \\ \left(0, \frac{1}{2}\right) & : \text { Möbius strip }\end{cases}
$$

Since the difference between planar and non-planar cylinders and the Möbius strip amounts to a constant shift of its arguments, $G_{p q}$ in (3.20) satisfies a universal differential equation,

$$
4 \pi\left(\frac{\partial G_{p q}}{\partial t}+\frac{\nu_{p q}}{t} \frac{\partial G_{p q}}{\partial \nu_{p}}\right)=-\left(\frac{\partial G_{p q}}{\partial \nu_{p}}\right)^{2}-\frac{\partial^{2} G_{p q}}{\partial \nu_{p}^{2}}+c(t)
$$

On the right hand side, the definition $(2.32)$ of $f_{p q}^{(2)}$ has been rewritten in terms of $\nu$ derivatives of $G_{p q}$. The function $c(t)$ in (3.21) does not depend on $\nu_{p}$ and will therefore 
drop out from the later discussion. The differential operator on the left hand side can be recognized as a derivative ${ }^{4}$ in the Jacobi transformed modular parameter:

$$
t^{\prime} \equiv \frac{1}{t}, \quad \nu^{\prime} \equiv \frac{\nu}{t} \quad \Rightarrow \quad \frac{\partial}{\partial t}+\frac{\nu_{p q}}{t} \frac{\partial}{\partial \nu_{p}}=-\left(t^{\prime}\right)^{2} \frac{\partial}{\partial t^{\prime}} .
$$

The original modular parameter $t$ can be interpreted as the circumference of the cylinder or the worldline length in the field-theory limit. ${ }^{5}$ Its Jacobi transform $t^{\prime}$, on the other hand, describes the length of the cylinder or the proper time in the closed-string channel. Analogous statements hold for the Möbius strip.

From (3.21) and (3.22), one can derive a universal relation for $f_{p q}^{(2)}$ analogous to (3.17),

$$
f^{(2)}\left(i \nu_{p q}+\delta \mid i t+\varepsilon\right)=-2 \pi\left(t^{\prime}\right)^{2} \frac{\partial}{\partial t^{\prime}} G\left(i \nu_{p q}+\delta \mid i t+\varepsilon\right)+\frac{c(t)}{2} .
$$

This allows to rewrite the $t^{\prime}$ derivative of the Koba-Nielsen factor (2.30) in terms of $f_{i j}^{(2)}$,

$$
\frac{\partial}{\partial t^{\prime}} \mathcal{I}\left(s_{i j}\right)=-\frac{\alpha^{\prime}}{2 \pi\left(t^{\prime}\right)^{2}} \mathcal{I}\left(s_{i j}\right) \sum_{i<j}^{6} s_{i j} f_{i j}^{(2)}
$$

which is valid for all topologies and where $c(t)$ in (3.23) cancels by momentum conservation $\sum_{i<j}^{6} s_{i j}=0$. Moreover, the pattern of Mandelstam variables and $f_{i j}^{(2)}$ on the right hand side reproduces the anomalous BRST transformation (3.9) of the six point correlator,

$$
Q\left(\mathcal{I}\left(s_{i j}\right) \mathcal{K}_{6}\right)=V_{1} \mathcal{Y}_{2,3,4,5,6} \frac{2 \pi\left(t^{\prime}\right)^{2}}{\alpha^{\prime}} \frac{\partial}{\partial t^{\prime}} \mathcal{I}\left(s_{i j}\right)
$$

Together with the Jacobi transformed integration measure $\mathrm{d} t=-\frac{\mathrm{d} t^{\prime}}{\left(t^{\prime}\right)^{2}}$, one can finally identify the BRST anomaly of the six point amplitude in (3.1) as a boundary term in $t^{\prime}$ :

$$
Q A_{6}^{\text {top }}=\frac{2 \pi}{\alpha^{\prime}} V_{1} \mathcal{Y}_{2,3,4,5,6} \int_{0}^{\infty} \mathrm{d} t^{\prime} \frac{\partial}{\partial t^{\prime}} \int_{\Delta_{\text {top }}} \mathrm{d} z_{2}^{\prime} \mathrm{d} z_{3}^{\prime} \ldots \mathrm{d} z_{6}^{\prime} \mathcal{I}\left(s_{i j}\right)
$$

where the transformation $\mathrm{d} z_{j}=i t \mathrm{~d} z_{j}^{\prime}$ has compensated for the factor of $t^{-5}$ in (2.30). Note that modular invariance of the Koba-Nielsen factor allows to collectively replace $G\left(i \nu_{p q} \mid i t\right) \rightarrow G\left(\nu_{p q}^{\prime} \mid i t^{\prime}\right)$. By the universality of (3.23), this analysis is valid for all topologies of open-string worldsheets and the anomaly is canceled for the gauge group $\mathrm{SO}(32)[21,22]$.

\section{The complete six-point amplitude of the closed string}

This section is devoted to the six-point one-loop amplitude among massless closed-string states of type IIA/IIB superstring theories. Before presenting the six-point function we revisit the five-point amplitude result of [37] to rewrite its kinematics in a factorized form.

\footnotetext{
${ }^{4}$ The partial derivative w.r.t. $t^{\prime}$ is understood to be evaluated at constant $\nu^{\prime}$.

${ }^{5} \mathrm{~A}$ pure spinor description of the six-point one-loop amplitude in ten-dimensional SYM including its hexagon anomaly can be found in [8].
} 


\subsection{The one-loop five point function for closed strings}

In [37] the pure spinor representation of the five-point closed-string amplitude in both type IIA/IIB was obtained ${ }^{6}$ (in the type IIA the chirality of the right-movers is reversed)

$$
\mathcal{M}_{5}=\int \frac{\mathrm{d}^{2} \tau}{\tau_{2}^{5}} \int \mathrm{d}^{2} z_{2} \ldots \mathrm{d}^{2} z_{5} \mathcal{I}\left(s_{i j}\right)\left(\mathcal{K}_{5} \tilde{\mathcal{K}}_{5}+\frac{\pi}{\tau_{2}} \mathcal{L}_{5}\right)
$$

where $\mathcal{K}_{5}$ is the open-string five point correlator and $\mathcal{L}_{5}$ encodes the interactions between the left- and right-movers (marked with tilde),

$$
\begin{aligned}
\mathcal{K}_{5}= & X_{23} C_{1 \mid 23,4,5}+(2,3 \mid 2,3,4,5), \\
\mathcal{L}_{5}= & M_{1} M_{2,3,4,5}^{m} \tilde{M}_{1} \tilde{M}_{2,3,4,5}^{m}+\left[s_{12} M_{12} M_{3,4,5} \tilde{M}_{12} \tilde{M}_{3,4,5}+(2 \leftrightarrow 3,4,5)\right] \\
& +\left[s_{23} M_{1} M_{23,4,5} \tilde{M}_{1} \tilde{M}_{23,4,5}-s_{23} C_{1 \mid 23,4,5} \tilde{C}_{1 \mid 23,4,5}+(2,3 \mid 2,3,4,5)\right],
\end{aligned}
$$

see (2.16) and (2.18) for the definitions of $M_{A, B, C}, M_{A, B, C, D}^{m}$ and (2.21) for $C_{1 \mid A, B, C}$ with

$$
\left\langle C_{1 \mid 23,4,5}\right\rangle=s_{45}\left[s_{24} A^{\mathrm{YM}}(1,3,2,4,5)-s_{34} A^{\mathrm{YM}}(1,2,3,4,5)\right] .
$$

The characteristic coefficient $\frac{\pi}{\tau_{2}}$ signals the mixing between left- and right-movers and arises from either the contraction $\Pi^{m}(z) \bar{\Pi}^{n}(\bar{z})$ or from left-moving derivatives acting on right-moving propagators in integration by parts identities,

$$
\Pi^{m}\left(z_{i}\right) \bar{\Pi}^{n}\left(\bar{z}_{j}\right) \rightarrow \delta^{m n} \frac{\pi}{\tau_{2}}, \quad \partial_{i} \bar{f}_{i j}^{(1)}=-\frac{\pi}{\tau_{2}} .
$$

While the amplitude (4.1) is BRST invariant the kinematic factor $\mathcal{L}_{5}$ is not manifestly BRST closed. However, by adding terms to $\mathcal{L}_{5}$ that vanish in the cohomology one arrives at a manifestly BRST invariant expression (the vector $C_{1 \mid 2,3,4,5}^{m}$ is reviewed ${ }^{7}$ in section 2.3),

$$
\mathcal{L}_{5}+\left[Q D_{1|2| 3,4,5} \tilde{M}_{12} \tilde{M}_{3,4,5}+M_{12} M_{3,4,5} \tilde{Q} \tilde{D}_{1|2| 3,4,5}+(2 \leftrightarrow 3,4,5)\right]=C_{1 \mid 2,3,4,5}^{m} \tilde{C}_{1 \mid 2,3,4,5}^{m}
$$

where (note that $\left\langle\mathcal{Y}_{1,2,3,4,5}\right\rangle=0$ in the five-particle momentum phase space) [26],

$$
\begin{aligned}
D_{1|2| 3,4,5} & \equiv \mathcal{J}_{2 \mid 1,3,4,5}+k_{2}^{m} M_{12,3,4,5}^{m}+\left[s_{23} M_{123,4,5}+(3 \leftrightarrow 4,5)\right], \\
Q D_{1|2| 3,4,5} & =\mathcal{Y}_{1,2,3,4,5}+k_{2}^{m} M_{1} M_{2,3,4,5}^{m}-s_{12} M_{12} M_{3,4,5}+\left[M_{1} M_{23,4,5}+(3 \leftrightarrow 4,5)\right] .
\end{aligned}
$$

Therefore the five-point amplitude (4.3) becomes

$$
\mathcal{M}_{5}=\int \frac{\mathrm{d}^{2} \tau}{\tau_{2}^{5}} \int \mathrm{d}^{2} z_{2} \ldots \mathrm{d}^{2} z_{5} \mathcal{I}\left(s_{i j}\right)\left\{\mathcal{K}_{5} \tilde{\mathcal{K}}_{5}+\frac{\pi}{\tau_{2}} C_{1 \mid 2,3,4,5}^{m} \tilde{C}_{1 \mid 2,3,4,5}^{m}\right\}
$$

up to the $Q$-exact terms in (4.6) that do not contribute upon zero-mode integration. This representation is manifestly $\mathrm{BRST}$ invariant (since $Q C_{1 \mid A, B, C}=Q C_{1 \mid A, B, C, D}^{m}=0$ ) and organizes the kinematic dependence in a factorized form w.r.t. left- and right-movers.

\footnotetext{
${ }^{6}$ The RNS and GS representations can be found in [38] and [30], respectively.

${ }^{7}$ The shorthand $C_{1 \mid 2,3,4,5}^{m} \tilde{C}_{1 \mid 2,3,4,5}^{m}$ was assigned a different meaning in [37] and differs from the right-hand side of (4.6) by $s_{23} C_{1 \mid 23,4,5} \tilde{C}_{1 \mid 23,4,5}+(2,3 \mid 2,3,4,5)$.
} 
The compactness and manifest BRST invariance of (4.8) demonstrate the virtue of vectorial BRST invariants to describe closed-string amplitudes. From the five-point example, one can anticipate that BRST (pseudo-)invariants of rank $r$ find a natural appearance in closed-string amplitudes at higher multiplicity $r+4$, along with $r$ powers of $\frac{\pi}{\tau_{2}}$. In the subsequent, this expectation is confirmed for the six-point amplitude.

\subsection{The six-point closed-string correlator}

The six-point closed-string correlator $\mathcal{M}_{6}$ combines the doubling of its open-string counterpart $\mathcal{K}_{6}=\mathcal{K}_{6}^{C}+\mathcal{K}_{6}^{P}$ with an extended set of left-right interactions,

$$
\mathcal{M}_{6}=\int \frac{\mathrm{d}^{2} \tau}{\tau_{2}^{5}} \int \mathrm{d}^{2} z_{2} \ldots \mathrm{d}^{2} z_{6} \mathcal{I}\left(s_{i j}\right)\left\{\mathcal{K}_{6} \tilde{\mathcal{K}}_{6}+\frac{\pi}{\tau_{2}} \mathcal{K}_{6}^{m} \tilde{\mathcal{K}}_{6}^{m}+\left(\frac{\pi}{\tau_{2}}\right)^{2} \mathcal{L}_{6}\right\}
$$

where (see (3.4) and (3.7) for the expressions of $\mathcal{K}_{6}^{C}$ and $\mathcal{K}_{6}^{P}$ )

$$
\begin{aligned}
\mathcal{K}_{6} & =\mathcal{K}_{6}^{C}+\mathcal{K}_{6}^{P} \\
\mathcal{K}_{6}^{m} & =X_{23} C_{1 \mid 23,4,5,6}^{m}+(2,3 \mid 2,3,4,5,6) .
\end{aligned}
$$

Note that $\mathcal{K}_{6}^{m}$ resembles the five-point open string correlator (4.2) where the scalar invariants $C_{1 \mid 23,4,5}$ are replaced by their vector counterparts $(2.22)$. The appearance of $\mathcal{K}_{6}^{m} \tilde{\mathcal{K}}_{6}^{m}$ has been carefully checked by keeping track of all the sources of $\frac{\pi}{\tau_{2}}$ shown in (4.5).

Finally, the kinematic factor $\mathcal{L}_{6}$ along with the quadratic piece $\frac{\pi^{2}}{\tau_{2}^{2}}$ in (4.9) contains the two-tensor generalization of left-right contractions supplemented by a quadratic expression of the pseudo-invariants (2.27),

$$
\mathcal{L}_{6}=\frac{1}{2} C_{1 \mid 2,3,4,5,6}^{m n} \tilde{C}_{1 \mid 2,3,4,5,6}^{m n}-\left[P_{1|2| 3,4,5,6} \tilde{P}_{1|2| 3,4,5,6}+(2 \leftrightarrow 3,4,5,6)\right] .
$$

The pseudo-invariants in (4.11) obstruct a representation of $\mathcal{L}_{6}$ as a tensor contraction of the form $\mathcal{K}_{6}^{m n} \tilde{\mathcal{K}}_{6}^{m n}$ but their presence compensates the anomalous BRST transformation (2.28) of the tensor $C_{1 \mid 2,3,4,5,6}^{m n}$ such that $Q \mathcal{L}_{6}=0$. In addition, we will show in the next section that the form of (4.11) is fixed by the low-energy limit.

Since the functions $f^{(n)}$ and $\tau_{2}^{-1}$ have modular weight $(n, 0)[28]$ and $(1,1)$, respectively, $\mathcal{K}_{6}$ and $\mathcal{K}_{6}^{m}$ carry modular weight $(2,0)$ and $(1,0)$ such that the expression in (4.9) manifests modular invariance of the closed-string amplitude.

\subsection{Low-energy limits and S-duality of type IIB}

In this subsection, we discuss the low-energy limit of one-loop amplitudes among massless closed-string states and relate it to the S-duality implications in type IIB theory.

As explained in $[30,37,39,40]$, the momentum dependence of torus integrals of the form (4.8) and (4.9) can be split into analytic and non-analytic contributions. ${ }^{8}$ The leading analytic behavior $\alpha^{\prime} \rightarrow 0$ follows unambiguously by setting $\mathcal{I}\left(s_{i j}\right) \rightarrow 1$ after taking the

\footnotetext{
${ }^{8}$ The interplay of the analytic and non-analytic parts of the amplitude as well as subtle ambiguities at higher $\alpha^{\prime}$ order and their resolution are discussed in [40].
} 
kinematic poles due to integration over $\mathrm{d}^{2} z \mathcal{I}\left(s_{i j}\right) \partial G_{23} \bar{\partial} G_{23} \rightarrow 2 \pi \mathrm{d} r_{23} r_{23}^{\alpha^{\prime} s_{23}-1}$ with $r_{23} \equiv$ $\left|z_{23}\right|$ into account, see e.g. [30, 37]. This gives rise to low-energy limits

$$
\begin{aligned}
\left.\mathcal{M}_{4}\right|_{\alpha^{\prime} \rightarrow 0}= & \left|C_{1 \mid 2,3,4}\right|^{2} \\
\left.\mathcal{M}_{5}\right|_{\alpha^{\prime} \rightarrow 0}= & {\left[s_{23}\left|C_{1 \mid 23,4,5}\right|^{2}+(2,3 \mid 2,3,4,5)\right]+\left|C_{1 \mid 2,3,4,5}^{m}\right|^{2} } \\
\left.\mathcal{M}_{6}\right|_{\alpha^{\prime} \rightarrow 0}= & {\left[s_{23}\left|C_{1 \mid 23,4,5,6}^{m}\right|^{2}+(2,3 \mid 2,3,4,5,6)\right]+\mathcal{L}_{6} } \\
& +\left[s_{23} s_{45}\left|C_{1 \mid 23,45,6}\right|^{2}+s_{24} s_{35}\left|C_{1 \mid 24,35,6}\right|^{2}+s_{25} s_{34}\left|C_{1 \mid 25,34,6}\right|^{2}+(6 \leftrightarrow 5,4,3,2)\right] \\
& +\left[s_{23} s_{34}\left|C_{1 \mid 234,5,6}\right|^{2}+s_{24} s_{43}\left|C_{1 \mid 243,5,6}\right|^{2}+s_{23} s_{24}\left|C_{1 \mid 324,5,6}\right|^{2}+(2,3,4 \mid 2,3,4,5,6)\right]
\end{aligned}
$$

with the shorthand notation $\left|C_{1 \mid 23,4,5,6}^{m}\right|^{2} \equiv C_{1 \mid 23,4,5,6}^{m} \tilde{C}_{1 \mid 23,4,5,6}^{m}$ and obvious generalizations. In the six-point case, the expressions (3.4) and (4.10) for $\mathcal{K}_{6}^{C}$ and $\mathcal{K}_{6}^{m}$ have been inserted into (4.9), whereas the expression (4.11) for $\mathcal{L}_{6}$ is treated as unknown at this point and will be derived in the subsequent. Note that the anomalous part $\mathcal{K}_{6}^{P}$ of the open-string correlator does not contribute to the low-energy limit due to the non-singular nature of the $f_{i j}^{(2)}$ as $z_{i} \rightarrow z_{j}$, see the discussion below (2.32).

At four and five points, the type IIB graviton components of (4.12) and (4.13) are proportional to the $\alpha^{\prime 3}$ order of tree-level amplitudes [37, 41]. They originate from the $R^{4}$ operator in the type IIB low-energy effective action whose tensor structure is determined by supersymmetry and whose coefficient (determined by S-duality) is given by the nonholomorphic Eisenstein series $E_{3 / 2}$ [42-44]. The non-linear extension of $R^{4}$ equally affects multiparticle amplitudes at the $\alpha^{\prime 3}$ order at tree level and in the low-energy limit at one loop and leads to the following S-duality prediction,

$$
\begin{aligned}
\left\langle\mathcal{M}_{N}||_{\alpha^{\prime} \rightarrow 0}^{\mathrm{IB}}\right\rangle= & c_{q} \sum_{\sigma, \rho \in S_{N-3}} A^{\mathrm{YM}}(1, \sigma(2,3, \ldots, N-2), N, N-1) \\
& \times\left(S_{0} M_{3}\right)_{\sigma, \rho} \tilde{A}^{\mathrm{YM}}(1, \rho(2,3, \ldots, N-2), N-1, N)
\end{aligned}
$$

whose proportionality constant $c_{q}$ does not depend on the multiplicity $N$. The right-hand side borrows the notation of [45] for the low-energy expansion of tree-level amplitudes involving $N$ closed-string states. The entries of the $(N-3) ! \times(N-3)$ ! matrices $S_{0}$ and $M_{3}$ are polynomials of degree $N-3$ and 3 in the dimensionless Mandelstam invariants $\alpha^{\prime}\left(k_{i} \cdot k_{j}\right)$, and $S_{0}$ is the momentum kernel [46] which appears in the field-theory limit of the KLT formula [47, 48]. The matrix $M_{3}$ captures the $\alpha^{\prime 3}$ order in the low-energy expansion of genus-zero worldsheet integrals. ${ }^{9}$

A slightly modified argument applies to the components of (4.15) which violate the $\mathrm{U}(1)$ R-symmetry of type IIB supergravity. This is indicated by the subscript $q$ of the proportionality constant $c_{q}$ in (4.15). The simplest non-vanishing amplitude with $\mathrm{U}(1)$ violation occurs at multiplicity five and charge $q= \pm 2$, involving for instance four gravitons

\footnotetext{
${ }^{9}$ The explicit form of these matrices for multiplicity $N \leq 7$ and the building blocks for $N=8,9$ can be downloaded from [49]. Initially addressed via hypergeometric functions [50, 51], the $\alpha^{\prime}$-corrections at tree level for any multiplicity can be recursively generated from the Drinfeld associator [52]. The organization of these integrals in $(N-3) ! \times(N-3)$ ! matrices has been essential to reveal the structure of the $\alpha^{\prime}-$ expansion [45], see [53] for relations to the associator.
} 
and one axio-dilaton, see $[37,54,55]$ for its $\alpha^{\prime}$-expansion. It was argued via S-duality and confirmed through explicit calculation that the constants in (4.15) for charges $q=0, \pm 2$ are related by $c_{ \pm 2}=-\frac{1}{3} c_{0}$ [37]. An analogous discussion of the low-energy limit of two-loop five-point amplitudes and their dependence on $\mathrm{R}$ charges can be found in [6].

Since the coefficient $\mathcal{L}_{6}$ of $\frac{\pi^{2}}{\tau_{2}^{2}}$ in the six-graviton amplitude (4.9) contributes to the low-energy limit (4.14), it can be determined from the S-duality prediction (4.15). More precisely, the form of $\mathcal{L}_{6}$ in (4.11) is inferred from the following reasoning.

The double contraction $\Pi^{m} \Pi^{n} \bar{\Pi}^{p} \bar{\Pi}^{q} \rightarrow 2 \delta^{m(p} \delta^{q) n}\left(\frac{\pi}{\tau_{2}}\right)^{2}$ gives rise to a contribution of the form $\frac{1}{2} M_{1} M_{2,3,4,5,6}^{m n} \tilde{M}_{1} \tilde{M}_{2,3,4,5,6}^{m n}$ whose unique BRST pseudo-invariant completion is given by $\mathcal{L}_{6}=\frac{1}{2} C_{1 \mid 2,3,4,5,6}^{m n} \tilde{C}_{1 \mid 2,3,4,5,6}^{m n}+\cdots[26]$. However, the BRST variation (2.28) and the trace relation $\delta_{m n} \tilde{C}_{1 \mid 2,3,4,5,6}^{m n}=2 \tilde{P}_{1|2| 3,4,5,6}+(2 \leftrightarrow 3,4,5,6)[26]$ yield

$$
Q C_{1 \mid 2,3,4,5,6}^{m n} \tilde{C}_{1 \mid 2,3,4,5,6}^{m n}=-2 V_{1} \mathcal{Y}_{2,3,4,5,6}\left[\tilde{P}_{1|2| 3,4,5,6}+(2 \leftrightarrow 3,4,5,6)\right] .
$$

Now the S-duality prediction relates the low-energy limit of the closed-string amplitude and the tree-level $\alpha^{\prime 3}$ terms via (4.15). Demanding the low-energy limit to be BRST invariant and permutation symmetric ${ }^{10}$ uniquely fixes $\mathcal{L}_{6}$ to the form (4.11). A component evaluation for six external gravitons confirms the matching with the tree-level amplitude at order $\alpha^{\prime 3}$.

\subsection{The BRST variation as a boundary term}

It will be demonstrated in this section ${ }^{11}$ that the BRST (or gauge) variation of the closedstring amplitude (4.9) gives rise to a total derivative in moduli space.

BRST invariance of $\mathcal{K}_{6}^{m}$ and $\mathcal{L}_{6}$ implies that

$$
Q \mathcal{M}_{6}=-V_{1} \mathcal{Y}_{2,3,4,5,6} \int \frac{\mathrm{d}^{2} \tau}{\tau_{2}^{5}} \int \mathrm{d}^{2} z_{2} \ldots \mathrm{d}^{2} z_{6} \mathcal{I}\left(s_{i j}\right) \sum_{i<j}^{6} s_{i j} f_{i j}^{(2)} \tilde{\mathcal{K}}_{6} .
$$

Using the representation (3.17) of the $f_{i j}^{(2)}$ function, the factor of $\sum_{i<j}^{6} s_{i j} f_{i j}^{(2)}$ can be expressed in terms of derivatives of the Koba-Nielsen factor with respect to $\tau$ and $z_{j}$ :

$$
\begin{aligned}
\frac{1}{2 \pi i} \mathcal{I}\left(s_{i j}\right) \sum_{i<j}^{6} s_{i j} f_{i j}^{(2)} & =\mathcal{I}\left(s_{i j}\right)\left(\sum_{i<j}^{6} s_{i j} \frac{\partial}{\partial \tau} G_{i j}+\frac{1}{\tau_{2}} \sum_{i=1}^{6} \operatorname{Im} z_{i} \sum_{j \neq i}^{6} s_{i j} \partial G_{i j}\right) \\
& =\frac{2}{\alpha^{\prime}}\left(\frac{\partial}{\partial \tau}+\sum_{p=2}^{6} \frac{\operatorname{Im} z_{p 1}}{\tau_{2}} \partial_{p}\right) \mathcal{I}\left(s_{i j}\right) .
\end{aligned}
$$

The second step is based on translation invariance $\partial_{1} \mathcal{I}=-\sum_{j=2}^{6} \partial_{j} \mathcal{I}$. It turns out that the differential operator in (4.19) annihilates the right-moving correlator $\tilde{\mathcal{K}}_{6}$ since [28]

$$
\partial \bar{f}_{i j}^{(k)}=-\frac{\pi}{\tau_{2}} \bar{f}_{i j}^{(k-1)}, \quad \frac{\partial \bar{f}_{i j}^{(k)}}{\partial \tau}=\frac{\pi \operatorname{Im} z_{i j}}{\tau_{2}^{2}} \bar{f}_{i j}^{(k-1)}, \quad k=1,2
$$

\footnotetext{
${ }^{10}$ Demonstrating permutation invariance of (4.14) with $\mathcal{L}_{6}$ given by (4.11) requires the canonicalization techniques in section 11 of [26].

${ }^{11}$ We are grateful to Michael Green for fruitful discussions which led to the results of this section.
} 
with $\bar{f}_{i j}^{(0)} \equiv 1$ imply that its constituents $\bar{f}_{i j}^{(2)}$ and $\bar{f}_{i j}^{(1)} \equiv \bar{\partial} G_{i j}$ satisfy

$$
\left(\frac{\partial}{\partial \tau}+\sum_{p=2}^{6} \frac{\operatorname{Im} z_{p 1}}{\tau_{2}} \partial_{p}\right) \bar{f}_{i j}^{(k)}=0 \quad k=1,2
$$

Hence, the BRST variation in (4.17) can be rewritten as

$$
Q \mathcal{M}_{6}=-\frac{4 \pi i}{\alpha^{\prime}} V_{1} \mathcal{Y}_{2,3,4,5,6} \int \frac{\mathrm{d}^{2} \tau}{\tau_{2}^{5}} \int \mathrm{d}^{2} z_{2} \ldots \mathrm{d}^{2} z_{6}\left(\frac{\partial}{\partial \tau}+\sum_{p=2}^{6} \frac{\operatorname{Im} z_{p 1}}{\tau_{2}} \partial_{p}\right)\left(\mathcal{I}\left(s_{i j}\right) \tilde{\mathcal{K}}_{6}\right)
$$

In order to identify this as total derivatives, we have to commute the differential operators $\frac{\partial}{\partial \tau}$ and $\partial_{p}$ past the factors of $\frac{1}{\tau_{2}^{5}}$ and $\frac{\operatorname{Im} z_{p 1}}{\tau_{2}}$, respectively. The commutators

$$
\left[\frac{1}{\tau_{2}^{5}}, \frac{\partial}{\partial \tau}\right]=-\frac{5 i}{2 \tau_{2}^{6}}, \quad\left[\frac{\operatorname{Im} z_{p 1}}{\tau_{2}^{6}}, \partial_{p}\right]=\frac{i}{2 \tau_{2}^{6}}
$$

mutually cancel after summing $p$ over $2,3, \ldots, 6$, so we conclude ${ }^{12}$ that the BRST variation of the six point function is a surface term in both $\tau$ and $z_{p}$ :

$$
\begin{aligned}
Q \mathcal{M}_{6}= & -\frac{4 \pi i}{\alpha^{\prime}} V_{1} \mathcal{Y}_{2,3,4,5,6} \int \mathrm{d}^{2} \tau\left\{\frac{\partial}{\partial \tau} \frac{1}{\tau_{2}^{5}} \int \mathrm{d}^{2} z_{2} \ldots \mathrm{d}^{2} z_{6} \mathcal{I}\left(s_{i j}\right) \tilde{\mathcal{K}}_{6}\right. \\
& \left.+\sum_{p=2}^{6} \int \mathrm{d}^{2} z_{2} \ldots \mathrm{d}^{2} z_{6} \partial_{p} \frac{\operatorname{Im} z_{p 1}}{\tau_{2}^{6}} \mathcal{I}\left(s_{i j}\right) \tilde{\mathcal{K}}_{6}\right\}
\end{aligned}
$$

The surface integral over the vertex insertions in the second line vanishes because the torus has no boundaries while the vanishing of the surface integral on moduli space follows from modular invariance [56-58].

\section{Conclusion and outlook}

In this work we combined the one-loop cohomology analysis of [26] with the worldsheet functions studied in [28] to write down the complete six-point one-loop amplitudes of the open and closed string. In doing so, we supplemented the BRST-invariant six-point correlator (3.4) that captures the worldsheet singularities among the external vertices with the non-singular pseudo-invariant correlator (3.7). The pseudo BRST invariance allows us to describe the hexagon gauge anomaly in pure spinor superspace while the non-singular worldsheet functions capture the regular parts of the correlator. Their composition given in (3.7) is such that its non-vanishing gauge variation (3.9) gives rise to a total derivative in moduli space (leading to the usual mechanism of anomaly cancellation $[21,22,31]$ ). This condition fixes the superspace form of the anomaly-containing part of the open-string correlator (3.7) and reproduces the bosonic results from earlier analyses within the RNS framework $[15,16]$.

\footnotetext{
${ }^{12}$ The action of $\frac{\partial}{\partial \tau}$ on the $\tau$ dependent integration domain for $z_{j}$ drops out because the resulting boundary term $z_{j}=\tau$ is suppressed by the Koba-Nielsen factor.
} 
The (pseudo-)invariant vector and tensor building blocks from the open string allow for elegant representations for closed-string one-loop amplitudes. As elaborated in section 4, any basis integral of the closed string is accompanied by a manifestly (pseudo-)invariant kinematic factor quadratic in the open-string (pseudo-)invariants. In order to arrive at the novel six-point result, in addition to an OPE-driven derivation of the singular part of the correlator, we also used S-duality considerations to completely fix its regular terms. This organizing principle for closed-string one-loop amplitudes has a natural extension beyond maximal supersymmetry, see [59] for examples in orbifold compactifications.

While the results of this paper demonstrate the value of the (pseudo-)cohomology framework of [26], it is imperative to derive them from first principles within the pure spinor formalism. This endeavor is expected to require a more in-depth understanding of how the non-zero modes of the b-ghost contribute to the final expressions in analogy to the RNS supercurrent in appendix B.2. These contributions are currently poorly understood and give rise to difficulties in extending the results for higher-loop amplitudes in $[5,6]$ beyond their low-energy limits.

Furthermore, for gauge groups different than $\mathrm{SO}(32)$, additional boundary terms along the lines of [60] arise from regularizing the divergent modular integral. ${ }^{13}$ These boundary terms give rise to worry about additional BRST anomalies and ambiguities associated with the choice of the regulator $\mathcal{N}$ for the non-compact space of pure spinors [18]. Subtleties of this type are not addressed in this work, and their treatment in a manifestly supersymmetric formalism is left as an interesting open problem.

\section{Acknowledgments}

We are very grateful to Tim Adamo, Nathan Berkovits, Eduardo Casali, Yu-tin Huang, David Skinner, Stephan Stieberger and in particular Michael Green for inspiring discussions. CRM wishes to acknowledge support from NSF grant number PHY 1314311 and the Paul Dirac Fund. OS thanks Marcus Berg and Igor Buchberger for collaboration on related topics and the Institute of Advanced Studies in Princeton for kind hospitality during finalization of this work. The research leading to these results has received funding from the European Research Council under the European Community's Seventh Framework Programme (FP7/2007-2013)/ERC grant agreement no. [247252]. Also, this material is supported by the National Science Foundation under Grant No. PHY-1066293 and the hospitality of the Aspen Center for Physics.

\section{A Permutation behavior of the open-string correlator}

In this appendix, we derive the asymmetry (3.16) of the six-point correlator $\mathcal{K}_{6}^{P}$ from the prescription (2.1) for open-string amplitudes. It will be demonstrated that an exchange of the unintegrated vertex operator $V_{1} U_{2} \rightarrow V_{2} U_{1}$ yields a boundary term accompanied by the kinematic factor $\left\langle\mathcal{Y}_{12,3,4,5,6}\right\rangle$ in (2.23) with parity-odd bosonic components (3.12).

\footnotetext{
${ }^{13}$ We are grateful to Michael Green for enlightening discussions on this point.
} 
The key tool is the multiparticle version of the integrated vertex operator in (2.2),

$$
\mathcal{U}_{12} \equiv \partial \theta^{\alpha} \mathcal{A}_{\alpha}^{12}+\Pi^{m} \mathcal{A}_{m}^{12}+d_{\alpha} \mathcal{W}_{12}^{\alpha}+\frac{1}{2} N_{m n} \mathcal{F}_{12}^{m n}
$$

where the linearized superfields in $U_{j}$ are promoted to their multiparticle versions defined in section 2.2. As a consequence of their multiparticle equations of motion, the BRST variation $Q U=\partial V$ generalizes to [13]

$$
Q \mathcal{U}_{12}=V_{1} U_{2}-V_{2} U_{1}+\partial M_{12}
$$

When inserting the left hand side into the amplitude prescription (2.1) in the place of $V_{1} U_{2}$, the analysis of $\{Q, b\}$ around (2.5) can be repeated to show that

$$
\begin{gathered}
\sum_{\text {top }} G_{\text {top }} \int_{0}^{\infty} \mathrm{d} t \int_{\Delta_{\text {top }}} \mathrm{d} z_{2} \mathrm{~d} z_{3} \ldots \mathrm{d} z_{n}\left\langle\mathcal{N}(b, \mu)\left(Q \mathcal{U}_{12}\right) \prod_{j=3}^{n} U_{j}\left(z_{j}\right)\right\rangle \\
=-\sum_{\text {top }} G_{\text {top }} \int_{0}^{\infty} \mathrm{d} t \frac{\partial}{\partial t} \int_{\Delta_{\text {top }}} \mathrm{d} z_{2} \ldots \mathrm{d} z_{6}\left\langle\mathcal{N} \mathcal{U}_{12} \prod_{j=3}^{6} U_{j}\left(z_{j}\right)\right\rangle .
\end{gathered}
$$

After integrating $d_{\alpha_{1}} d_{\alpha_{2}} d_{\alpha_{3}} d_{\alpha_{4}} d_{\alpha_{5}} \rightarrow\left(\lambda \gamma^{m}\right)_{\alpha_{1}}\left(\lambda \gamma^{n}\right)_{\alpha_{2}}\left(\lambda \gamma^{p}\right)_{\alpha_{3}}\left(\gamma_{m n p}\right)_{\alpha_{4} \alpha_{5}}$ [24] for the only term $\left(d \mathcal{W}_{12}\right)\left(d W_{3}\right) \ldots\left(d W_{6}\right)$ with a sufficient number of $d_{\alpha}$ zero modes, (A.3) evaluates to

$$
\left\langle\mathcal{Y}_{12,3,4,5,6}\right\rangle \sum_{\text {top }} G_{\text {top }} \int_{0}^{\infty} \frac{\mathrm{d} t}{t^{5}} \int_{\Delta_{\text {top }}} \mathrm{d} z_{2} \ldots \mathrm{d} z_{6} \sum_{i<j}^{6} s_{i j} f_{i j}^{(2)} \mathcal{I}\left(s_{i j}\right)
$$

by the definition (2.23) of the anomaly superfield. Using the Jacobi-transformed variables $t^{\prime}=\frac{1}{t}$ and $z^{\prime}=\frac{z}{t}$ in intermediate steps, the modular derivative of the KobaNielsen factor $\mathcal{I}\left(s_{i j}\right)$ in (2.30) has been evaluated via (3.24) and gives rise to the functions $f_{i j}^{(2)}$ defined in (2.32). In the conventions of (3.1), one can read off the contribution of $\left\langle\mathcal{Y}_{12,3,4,5,6}\right\rangle \sum_{i<j}^{6} s_{i j} f_{i j}^{(2)}$ to the antisymmetric part of the kinematic factor $\mathcal{K}_{6}$ from (A.4).

This needs to be compared with the amplitude prescription (2.1) involving the right hand side of (A.2): the total derivative $\partial M_{12}$ decouples by the suppression of boundary terms in $z_{j}$ via $z_{i j}^{\alpha^{\prime}} s_{i j}$, and the leftover term $V_{1} U_{2}-V_{2} U_{1}$ yields the desired difference between $\mathcal{K}_{6}$ and its image under $(1 \leftrightarrow 2)$. This completes the proof of (3.16).

\section{B Comparison with the RNS computation}

We have checked the six-point open-string amplitude (3.7) in pure spinor superspace to reproduce the gluon amplitude from the RNS formalism upon component expansion [27]. Since this comparison rests on the availability of both expressions in a basis of worldsheet integrals, we will sketch the underlying integral reduction on the RNS side in this appendix. 


\section{B.1 The parity-even part}

The RNS prescription for the parity-even part of one-loop amplitudes is given by

$$
\begin{aligned}
A_{6, \text { even }}^{\text {top }} \sim & \int_{0}^{\infty} \mathrm{d} t \int_{\Delta_{\text {top }}} \mathrm{d} z_{2} \mathrm{~d} z_{3} \ldots \mathrm{d} z_{6} \sum_{\nu=1,2,3}(-1)^{\nu}\left(\frac{\theta_{\nu+1}(0, \tau)}{\theta_{1}^{\prime}(0, \tau)}\right)^{4} \\
& \times\left\langle V_{1}\left(e_{1}, k_{1}, z_{1}\right) V_{2}\left(e_{2}, k_{2}, z_{2}\right) \ldots V_{6}\left(e_{6}, k_{6}, z_{6}\right)\right\rangle_{\nu, \tau}
\end{aligned}
$$

where $V_{i}$ denotes the vertex operator of the gluon in the superghost picture zero:

$$
V_{1}\left(e_{1}, k_{1}, z_{1}\right) \equiv e_{1}^{m}\left[\partial x_{m}\left(z_{1}\right)+2 \alpha^{\prime} k_{1}^{n} \psi_{n} \psi_{m}\left(z_{1}\right)\right] e^{i k_{1} \cdot x\left(z_{1}\right)}
$$

The bracket $\langle\ldots\rangle_{\nu, \tau}$ instructs to evaluate the correlator in (B.1) on a genus-one Riemann surface with modular parameter $\tau$, and $\nu=1,2,3$ encode the even spin structures of the worldsheet spinors $\psi^{m}$ associated with partition functions $(-1)^{\nu}\left(\frac{\theta_{\nu+1}(0 \mid \tau)}{\theta_{1}^{\prime}(0 \mid \tau)}\right)^{4}[15]$.

Correlators among $x^{m}$ and $\psi^{m}$ can be straightforwardly evaluated using Wick contractions $x^{m}\left(z_{i}\right) x^{n}\left(z_{j}\right) \rightarrow-2 \alpha^{\prime} \delta^{m n} G_{i j}$ and $\psi^{m}\left(z_{i}\right) \psi^{n}\left(z_{j}\right) \rightarrow \delta^{m n} S_{\nu}\left(z_{i j} \mid \tau\right)$. The latter give rise to spin structure dependent Szegö kernels

$$
S_{\nu}(z \mid \tau) \equiv \frac{\theta_{1}^{\prime}(0 \mid \tau) \theta_{\nu+1}(z \mid \tau)}{\theta_{\nu+1}(0 \mid \tau) \theta_{1}(z \mid \tau)}
$$

with $\nu=1,2,3$. Together with the partition function in the first line of (B.1), the summation over spin structures can be described by the following building block

$$
\mathcal{G}_{n}\left(x_{1}, x_{2}, \ldots, x_{n} \mid \tau\right) \equiv \sum_{\nu=1,2,3}(-1)^{\nu}\left(\frac{\theta_{\nu+1}(0 \mid \tau)}{\theta_{1}^{\prime}(0 \mid \tau)}\right)^{4} S_{\nu}\left(x_{1} \mid \tau\right) S_{\nu}\left(x_{2} \mid \tau\right) \ldots S_{\nu}\left(x_{n} \mid \tau\right)
$$

with $x_{1}+x_{2}+\ldots+x_{n}=0$. As is well known, correlators with less than eight $\psi^{m}$ yield a vanishing spin sum, and $\mathcal{G}_{4}$ is the first instance where Riemann identities yield a nonvanishing result,

$$
\mathcal{G}_{n \leq 3}\left(x_{1}, x_{2}, \ldots, x_{n} \mid \tau\right)=0, \quad \mathcal{G}_{4}\left(x_{1}, x_{2}, x_{3}, x_{4} \mid \tau\right)=1,
$$

reflecting maximal spacetime supersymmetry. Representatives at multiplicity five and higher have been evaluated in $[15,34,35]$ using Fay trisecant identities in slightly different guises. The results of these references are equivalent to [28]

$$
\begin{aligned}
\mathcal{G}_{5}\left(x_{1}, x_{2}, x_{3}, x_{4}, x_{5} \mid \tau\right) & =\sum_{i=1}^{5} f_{i}^{(1)} \\
\mathcal{G}_{6}\left(x_{1}, x_{2}, x_{3}, x_{4}, x_{5}, x_{6} \mid \tau\right) & =\sum_{i=1}^{6} f_{i}^{(2)}+\sum_{i<j}^{6} f_{i}^{(1)} f_{j}^{(1)},
\end{aligned}
$$

where $f_{i}^{(k)} \equiv f^{(k)}\left(x_{i} \mid \tau\right)$ are defined by (2.31) and (2.32), respectively.

In order to cast the RNS amplitude (B.1) into the same basis of integrals as seen in the expression (3.4) and (3.7) for $\mathcal{K}_{6}^{C}$ and $\mathcal{K}_{6}^{P}$ in pure spinor superspace, we organize the integral reduction into three steps: 
- (i) elimination of double derivatives: bilinears in $\partial x^{m}\left(z_{i}\right)$ from the vertex operator (B.2) contract to a double derivative $\partial^{2} G_{i j}$ of the Green function (2.29). Since there is always a partner term $\alpha^{\prime} s_{i j}\left(\partial G_{i j}\right)^{2}$ with the same tensor structure from the fermionic part $\sim \psi^{2}$ of the vertex operators, the double pole of $\partial^{2} G_{i j},\left(\partial G_{i j}\right)^{2} \sim \frac{1}{z_{i j}^{2}}$ turns out to be spurious. This can be seen from a total derivative relation involving the Koba-Nielsen factor $\mathcal{I}$ from (2.30):

$$
\partial_{i}\left(\partial G_{i j} \mathcal{I}\right)=\left[\partial^{2} G_{i j}+\alpha^{\prime} s_{i j}\left(\partial G_{i j}\right)^{2}+\alpha^{\prime} \partial G_{i j} \sum_{p \neq i, j} X_{i p}\right] \mathcal{I}
$$

The residue of the double pole must be proportional to $\left(1-\alpha^{\prime} s_{i j}\right)$ since it would otherwise signal tachyon propagation.

- (ii) partial fraction relations: step (i) and (B.7) leave two topologies of bilinears in the propagator: $\partial G_{i j} \partial G_{i k}$ with $j \neq k$ and an overlapping leg $i$ as well as the disconnected configuration $\partial G_{i j} \partial G_{p q}$ with all of $i, j, p, q$ distinct. The former requires an application of the Fay identity (2.34) before the pattern of functions $X_{i j}\left(X_{i k}+X_{j k}\right)$ seen in (3.4) and suitable for step (iii) is manifest:

$$
\partial G_{i j} \partial G_{i k}=\frac{s_{j k}\left(f_{i j}^{(2)}+f_{i k}^{(2)}+f_{j k}^{(2)}\right)}{s_{i j k}}+\frac{X_{i j}\left(X_{i k}+X_{j k}\right)}{s_{i j} s_{i j k}}+\frac{X_{i k}\left(X_{i j}+X_{k j}\right)}{s_{i k} s_{i j k}} .
$$

- (iii) integration by parts: as explained below (3.3), the minimal set of worldsheet functions $X_{i j}$ is obtained by eliminating any instance of $X_{1 j}$ by discarding derivatives of the Koba-Nielsen factor (2.30) w.r.t. $z_{j}$. This amounts to two equivalent manipulations after step (ii):

$$
\begin{aligned}
X_{12}\left(X_{13}+X_{23}\right) & =\left(X_{23}+X_{24}+X_{25}+X_{26}\right)\left(X_{34}+X_{35}+X_{36}\right)+\partial_{2}(\ldots)+\partial_{3}(\ldots) \\
X_{12} X_{34} & =\left(X_{23}+X_{24}+X_{25}+X_{26}\right) X_{34}+\partial_{2}(\ldots)
\end{aligned}
$$

The $f_{i j}^{(2)}$ functions from (B.7) and step (ii) do not admit further simplification, in particular the five instances of $f_{1 j}^{(2)}$ cannot be reduced to $f_{p q}^{(2)}$ and $X_{p q}$ with $p, q \neq 1$. After performing the above steps, the agreement of bosonic components in the two formalisms,

$$
A_{6, \text { even }}^{\text {top }}=\left.\left\langle A_{6}^{\text {top }}\right\rangle\right|_{\text {parity-even }},
$$

can be checked along with each instance of $f_{i j}^{(2)}, X_{i j}\left(X_{i k}+X_{j k}\right)$ and $X_{i j} X_{p q}$, see (3.1) for the definition of the right hand side.

\section{B.2 The parity-odd part}

The parity-odd sector of the RNS six-point amplitude stems from the spin structure of $\psi^{m}$ with anti-periodic boundary conditions along both cycles of the Riemann surface. In 
this case, zero modes of the $\beta, \gamma$ ghosts as well as the ten components of $\psi^{m}$ have to be saturated in the path integral. This gives rise to the amplitude prescription

$$
\begin{aligned}
A_{6, \text { odd }}^{\text {top }} \sim & \frac{1}{2} \int_{0}^{\infty} \mathrm{d} t \int_{\Delta_{\text {top }}} \mathrm{d} z_{2} \mathrm{~d} z_{3} \ldots \mathrm{d} z_{6} \\
& \times\left\langle\partial x_{p}\left(z_{0}\right) \psi^{p}\left(z_{0}\right) \widehat{V}_{1}\left(e_{1}, k_{1}, z_{1}\right) V_{2}\left(e_{2}, k_{2}, z_{2}\right) \ldots V_{6}\left(e_{6}, k_{6}, z_{6}\right)\right\rangle_{\tau},
\end{aligned}
$$

where $\widehat{V}_{1}\left(e_{1}, k_{1}, z_{1}\right)$ denotes the gluon vertex operator in the picture of superghost charge -1 , and the zero mode integration for the $\beta, \gamma$ system has already been carried out [61]

$$
\widehat{V}_{1}\left(e_{1}, k_{1}, z_{1}\right) \equiv e_{1}^{m} \psi_{m}\left(z_{1}\right) e^{i k_{1} \cdot x\left(z_{1}\right)} .
$$

The worldsheet supercurrent $\partial x_{p} \psi^{p}$ is a remnant of a picture changing operator whose position $z_{0}$ drops out from the correlator. In the evaluation of the correlator (B.12), the Wick contraction of $\psi^{m}$ is adjusted to the spin structure $\psi^{m}\left(z_{i}\right) \psi^{n}\left(z_{j}\right) \rightarrow \delta^{m n} \partial G_{i j}$, and the zero mode integration amounts to absorbing

$$
\psi^{m_{1}}\left(w_{1}\right) \psi^{m_{2}}\left(w_{2}\right) \ldots \psi^{m_{10}}\left(w_{10}\right) \rightarrow \epsilon^{m_{1} m_{2} \ldots m_{10}},
$$

independently on $w_{j}$. After simplifying the parity-odd kinematic factors and eliminating the double derivatives of $G_{0 j}$ in a way similar to (B.8),

$$
\partial^{2} G_{0 j} \mathcal{I}\left(s_{i j}\right)=\alpha^{\prime} \mathcal{I}\left(s_{i j}\right) \partial G_{0 j} \sum_{p \neq j} X_{j p}+\partial_{j}(\ldots),
$$

we arrive at the following expression for (B.12):

$$
A_{6, \text { odd }}^{\text {top }}=\int_{0}^{\infty} \frac{\mathrm{d} t}{t^{5}} \int_{\Delta_{\text {top }}} \mathrm{d} z_{2} \ldots \mathrm{d} z_{6} \mathcal{I}\left(s_{i j}\right)\left\{\sum_{2 \leq p<q}^{6} \mathcal{E}_{p q}\left[\eta_{0 p q}-\eta_{01 p}-\eta_{01 q}-\left(\partial G_{01}\right)^{2}\right]\right\} \text {. }
$$

The worldsheet functions contained in $\eta_{i j k} \equiv \partial G_{i j} \partial G_{i k}+\operatorname{cyc}(i, j, k)$ can be rewritten as $f_{i j}^{(2)}+f_{j k}^{(2)}+f_{k i}^{(2)}$ via $(2.34)$, and the shorthand $\mathcal{E}_{p q}$ encoding the polarization dependence is defined by (permutations of)

$$
\mathcal{E}_{23} \equiv \frac{1}{2} e_{1}^{m}\left[\left(e_{2} \cdot k_{3}\right) k_{2}^{n}-s_{23} e_{2}^{n}\right] \epsilon_{m n r_{3} s_{3} \ldots r_{6} s_{6}} k_{3}^{r_{3}} e_{3}^{s_{3}} \ldots k_{6}^{r_{6}} e_{6}^{s_{6}}+(2 \leftrightarrow 3) .
$$

The sum of the ten inequivalent $\mathcal{E}_{p q}$ in (B.16) can be written as an antisymmetrization in eleven vector indices such that

$$
\sum_{2 \leq p<q} \mathcal{E}_{p q}=0
$$

This is crucial to cancel the contributions of $\partial G_{01}^{2}$ and $f_{01}^{(2)}$ in $\eta_{0 p q}-\eta_{01 p}-\eta_{01 q}-\left(\partial G_{01}\right)^{2}$ such that the position of the supercurrent in (B.12) drops out. We are left with

$$
A_{6, \text { odd }}^{\text {top }}=\int_{0}^{\infty} \frac{\mathrm{d} t}{t^{5}} \int_{\Delta_{\text {top }}} \mathrm{d} z_{2} \mathrm{~d} z_{3} \ldots \mathrm{d} z_{6} \mathcal{I}\left(s_{i j}\right)\left\{\sum_{2 \leq p<q}^{6} f_{p q}^{(2)} \mathcal{E}_{p q}+\sum_{p=2}^{6} f_{1 p}^{(2)} \mathcal{E}_{p}\right\},
$$


where the functions $f_{1 p}^{(2)}$ pick up polarization dependencies such as

$$
\mathcal{E}_{2} \equiv-\sum_{q=3}^{6} \mathcal{E}_{2 q}=\frac{1}{2}\left[\left(e_{1} \cdot k_{2}\right) k_{2}^{m} e_{2}^{n}+\left(e_{2} \cdot k_{1}\right) k_{1}^{m} e_{1}^{n}-s_{12} e_{1}^{m} e_{2}^{n}\right] \epsilon_{m n r_{3} s_{3} \ldots r_{6} s_{6}} k_{3}^{r_{3}} e_{3}^{s_{3}} \ldots k_{6}^{r_{6}} e_{6}^{s_{6}}
$$

along with $f_{12}^{(2)}$. The kinematic factors (B.17) and (B.20) are easily seen to be gauge invariant w.r.t. $e_{j}^{m} \rightarrow k_{j}^{m}$ for $j \neq 1$. However, the variation $e_{1}^{m} \rightarrow k_{1}^{m}$ in the first leg (represented by the vertex operator (B.13) of superghost picture -1) gives rise to

$$
\begin{aligned}
\left.\mathcal{E}_{p q}\right|_{e_{1}^{m} \rightarrow k_{1}^{m}} & =s_{p q} \times \epsilon_{m_{2} n_{2} \ldots m_{6} n_{6}} k_{2}^{m_{2}} e_{2}^{n_{2}} \ldots k_{6}^{m_{6}} e_{6}^{n_{6}} \\
\left.\mathcal{E}_{p}\right|_{e_{1}^{m} \rightarrow k_{1}^{m}} & =s_{1 p} \times \epsilon_{m_{2} n_{2} \ldots m_{6} n_{6}} k_{2}^{m_{2}} e_{2}^{n_{2}} \ldots k_{6}^{m_{6}} e_{6}^{n_{6}},
\end{aligned}
$$

since an $\epsilon$ contraction of all the six momenta $k_{1}, k_{2}, \ldots, k_{6}$ vanishes by momentum conservation. The resulting gauge anomaly

$$
\begin{aligned}
\left.A_{6, \text { odd }}^{\text {top }}\right|_{e_{1}^{m} \rightarrow k_{1}^{m}}= & \epsilon_{m_{2} n_{2} \ldots m_{6} n_{6}} k_{2}^{m_{2}} e_{2}^{n_{2}} \ldots k_{6}^{m_{6}} e_{6}^{n_{6}} \\
& \times \int_{0}^{\infty} \frac{\mathrm{d} t}{t^{5}} \int_{\Delta_{\text {top }}} \mathrm{d} z_{2} \mathrm{~d} z_{3} \ldots \mathrm{d} z_{6} \mathcal{I}\left(s_{i j}\right) \sum_{1 \leq p<q}^{6} s_{p q} f_{p q}^{(2)}
\end{aligned}
$$

is the fingerprint of the anomalous BRST variation (3.9) on the bosonic components, see appendix $\mathrm{C}$ for a superspace discussion of gauge variations. The parity-odd part (B.19) of the RNS amplitude agrees with the bosonic components of the superamplitude in (3.1),

$$
A_{6, \text { odd }}^{\text {top }}=\left.\left\langle A_{6}^{\text {top }}\right\rangle\right|_{\text {parity-odd }}
$$

which is found by comparing the coefficient of any $f_{i j}^{(2)}$.

\section{Gauge transformation versus BRST transformation}

In this appendix, it is demonstrated that linearized gauge transformations of the external states are encoded in the BRST variations of the kinematic factors. We thereby prove the equivalence of the anomalous BRST and gauge variations (3.9) of the six-point open-string amplitude (3.4) and (3.7).

\section{C.1 Gauge variation of multiparticle superfields}

The response of linearized SYM superfields to a superspace gauge transformation $\delta_{i}$ in particle $i$ is given by

$$
\delta_{i} A_{\alpha}^{i}=D_{\alpha} \Omega_{i}, \quad \delta_{i} A_{m}^{i}=k_{m}^{i} \Omega_{i}, \quad \delta_{i} W_{i}^{\alpha}=\delta_{i} F_{i}^{m n}=0,
$$

for scalar superfields $\Omega_{i}$, leading to the variations (2.3) of the massless vertex operators. For the choice $\Omega_{i}=e^{i k_{i} \cdot x}$, the gauge transformation (C.1) amounts to a transverse gluon polarization $e_{i}^{m} \rightarrow k_{i}^{m}$. 
The recursive construction of multiparticle superfields $\mathcal{A}_{\alpha}^{P}, \mathcal{A}_{P}^{m}, \mathcal{W}_{P}^{\alpha}, \mathcal{F}_{P}^{m n}$ in (2.9) to (2.13) determines their linearized gauge variation from (C.1). As pioneered in appendix B of [26] and generalized in [25], multiparticle gauge transformations are conveniently captured by multiparticle gauge scalars

$$
\mathcal{G}_{P} \equiv \frac{1}{s_{P}} \sum_{X Y=P}\left[\mathcal{G}_{Y}\left(k_{Y} \cdot \mathcal{A}_{X}\right)-\mathcal{G}_{X}\left(k_{X} \cdot \mathcal{A}_{Y}\right)\right]
$$

Performing a linearized gauge transformation (C.1) in a single external leg (say leg $i=1$ ) amounts to the initial condition $\mathcal{G}_{j}=\delta_{1, j} \Omega_{1}$ for the recursion (C.2), i.e. to having only one non-vanishing single-particle scalar $\mathcal{G}_{j}$. The induced gauge transformation of multiparticle superfields is given by [25]

$$
\begin{aligned}
\delta_{\mathcal{G}} \mathcal{A}_{\alpha}^{P} & =D_{\alpha} \mathcal{G}_{P}+\sum_{X Y=P}\left(\mathcal{G}_{X} \mathcal{A}_{\alpha}^{Y}-\mathcal{G}_{Y} \mathcal{A}_{\alpha}^{X}\right) \\
\delta_{\mathcal{G}} \mathcal{A}_{P}^{m} & =k_{P}^{m} \mathcal{G}_{P}+\sum_{X Y=P}\left(\mathcal{G}_{X} \mathcal{A}_{Y}^{m}-\mathcal{G}_{Y} \mathcal{A}_{X}^{m}\right) \\
\delta_{\mathcal{G}} \mathcal{W}_{P}^{\alpha} & =\sum_{X Y=P}\left(\mathcal{G}_{X} \mathcal{W}_{Y}^{\alpha}-\mathcal{G}_{Y} \mathcal{W}_{X}^{\alpha}\right) \\
\delta_{\mathcal{G}} \mathcal{F}_{P}^{m n} & =\sum_{X Y=P}\left(\mathcal{G}_{X} \mathcal{F}_{Y}^{m n}-\mathcal{G}_{Y} \mathcal{F}_{X}^{m n}\right)
\end{aligned}
$$

where the $\delta_{\mathcal{G}}$ operation reduces to the linearized variations (C.1) in any leg $i$ for appropriate choices of initial conditions. As a consequence, the one-loop building blocks in (2.15) to (2.19) transform as

$$
\begin{aligned}
\delta_{\mathcal{G}} M_{A}= & Q \mathcal{G}_{A}+\sum_{X Y=A}\left(\mathcal{G}_{X} M_{Y}-\mathcal{G}_{Y} M_{X}\right) \\
\delta_{\mathcal{G}} M_{A, B, C}= & \sum_{X Y=A}\left(\mathcal{G}_{X} M_{Y, B, C}-\mathcal{G}_{Y} M_{X, B, C}\right)+(A \leftrightarrow B, C) \\
\delta_{\mathcal{G}} M_{A, B, C, D}^{m}= & \sum_{X Y=A}\left(\mathcal{G}_{X} M_{Y, B, C, D}^{m}-\mathcal{G}_{Y} M_{X, B, C, D}^{m}\right) \\
& +k_{A}^{m} \mathcal{G}_{A} M_{B, C, D}+(A \leftrightarrow B, C, D) \\
\delta_{\mathcal{G}} M_{A, B, C, D, E}^{m n}= & \sum_{X Y=A}\left(\mathcal{G}_{X} M_{Y, B, C, D, E}^{m n}-\mathcal{G}_{Y} M_{X, B, C, D, E}^{m n}\right) \\
& +2 k_{A}^{(m} \mathcal{G}_{A} M_{B, C, D, E}^{m)}+(A \leftrightarrow B, C, D, E),
\end{aligned}
$$

and the anomaly current (2.25) exhibits the following gauge variation:

$$
\delta_{\mathcal{G}} J_{2 \mid 3,4,5,6}=k_{2}^{m} \mathcal{G}_{2} M_{3,4,5,6}^{m}+\left[s_{23} \mathcal{G}_{23} M_{4,5,6}+(3 \leftrightarrow 4,5,6)\right] .
$$

The multiparticle response to gauge variations can be conveniently interpreted by assembling both the gauge scalars $\mathcal{G}_{P}$ and the multiparticle superfields $\mathcal{A}_{\alpha}^{P}, \mathcal{A}_{m}^{P}, \ldots$ in a generating series: while latter solve the non-linear equations of motion of ten-dimensional SYM [62], the resummation of the gauge scalars encodes their non-linear gauge transformations. The recursion (C.2) is obtained by demanding the non-linear gauge transformations to preserve the Lorentz-gauge condition for the generating series of $\mathcal{A}_{m}^{P}$ [25]. The benefits of certain different choices of multiparticle gauge scalars are discussed in the reference. 


\section{C.2 Gauge variation of BRST (pseudo-)invariants}

For all of the kinematic building blocks $\left\{M_{A}, M_{A, B, C}, \ldots\right\}$ in the amplitudes under discussion, the BRST variations (2.20), (2.24) and (2.25) closely resemble the gauge variations (C.7) to (C.11). It is therefore not surprising that BRST invariants such as the scalars $C_{1 \mid A, B, C}$ and the vectors $C_{1 \mid A, B, C, D}^{m}$ in section 2.3 give rise to a $Q$-exact gauge variation

$$
\delta_{\mathcal{G}} C_{1 \mid A, B, C}=Q\left[\left.C_{1 \mid A, B, C}\right|_{M_{P} \rightarrow \mathcal{G}_{P}}\right], \quad \delta_{\mathcal{G}} C_{1 \mid A, B, C, D}^{m}=Q\left[\left.C_{1 \mid A, B, C, D}^{m}\right|_{M_{P} \rightarrow \mathcal{G}_{P}}\right],
$$

leading to vanishing components,

$$
\left\langle\delta_{\mathcal{G}} C_{1 \mid A, B, C}\right\rangle=0, \quad\left\langle\delta_{\mathcal{G}} C_{1 \mid A, B, C, D}^{m}\right\rangle=0 .
$$

For instance the five-point BRST invariant $C_{1 \mid 23,4,5}=M_{1} M_{23,4,5}+M_{12} M_{3,4,5}-M_{13} M_{2,4,5}$ translates into the gauge variation $\delta_{\mathcal{G}} C_{1 \mid 23,4,5}=Q\left(\mathcal{G}_{1} M_{23,4,5}+\mathcal{G}_{12} M_{3,4,5}-\mathcal{G}_{13} M_{2,4,5}\right)$ captured by the replacement $M_{P} \rightarrow \mathcal{G}_{P}$.

For the tensor $M_{A, B, C, D, E}^{m n}$ and the anomaly current $\mathcal{J}_{2 \mid 3,4,5,6}$, however, the superfields $\mathcal{Y}_{A, B, C, D, E}$ in their BRST variations (2.24) and (2.25) do not have any correspondent in the gauge variations (C.10) and (C.11). That is why the gauge transformation of their pseudo-invariant completions $C_{1 \mid 2,3,4,5,6}^{m n}$ and $P_{1|2| 3,4,5,6}$ in (2.26) and (2.27) exhibit anomalous admixtures

$$
\begin{aligned}
& \delta_{\mathcal{G}} C_{1 \mid 2,3,4,5,6}^{m n}=Q\left[\left.C_{1 \mid 2,3,4,5,6}^{m n}\right|_{M_{P} \rightarrow \mathcal{G}_{P}}\right]-\delta^{m n} \mathcal{G}_{1} \mathcal{Y}_{2,3,4,5,6} \\
& \delta_{\mathcal{G}} P_{1|2| 3,4,5,6}=Q\left[\left.P_{1|2| 3,4,5,6}\right|_{M_{P} \rightarrow \mathcal{G}_{P}}\right]-\mathcal{G}_{1} \mathcal{Y}_{2,3,4,5,6}
\end{aligned}
$$

The components $\left\langle\delta_{\mathcal{G}} C_{1 \mid 2,3,4,5,6}^{m n}\right\rangle$ and $\left\langle\delta_{\mathcal{G}} P_{1|2| 3,4,5,6}\right\rangle$ only depend on $\mathcal{G}_{1}$ whereas any other $\mathcal{G}_{j \neq 1}$ drops out. This reflects the initial observation in section 2.1 that gauge transformations of the integrated vertices $U_{2}, \ldots, U_{6}$ annihilate the six-point amplitude while the unintegrated vertex $V_{1}$ yields the anomaly (2.6) upon variation to $Q \Omega_{1}$. Setting $\mathcal{G}_{j} \rightarrow \delta_{j, 1} \Omega_{1} e^{i k_{j} x}$ reproduces the anomaly kinematic factor $K$ in $(2.7)$ :

$$
\left\langle\delta_{\mathcal{G}} C_{1 \mid 2,3,4,5,6}^{m n}\right\rangle \rightarrow-\frac{1}{2} \delta^{m n} K, \quad\left\langle\delta_{\mathcal{G}} P_{1|2| 3,4,5,6}\right\rangle \rightarrow-\frac{1}{2} K .
$$

The gauge anomaly in (C.14) and (C.15) obviously matches the anomalous BRST variations (2.28) upon adjusting $\mathcal{G}_{j} \rightarrow V_{j}$ in the non-exact part. Hence, the mechanism of anomaly cancellation is completely analogous for gauge and BRST transformations, see $[21,22]$ for open strings, and section 4.4 for the closed-string discussion.

Open Access. This article is distributed under the terms of the Creative Commons Attribution License (CC-BY 4.0), which permits any use, distribution and reproduction in any medium, provided the original author(s) and source are credited.

\section{References}

[1] N. Berkovits, Multiloop amplitudes and vanishing theorems using the pure spinor formalism for the superstring, JHEP 09 (2004) 047 [hep-th/0406055] [INSPIRE]. 
[2] N. Berkovits, Super-Poincaré covariant two-loop superstring amplitudes, JHEP 01 (2006) 005 [hep-th/0503197] [INSPIRE].

[3] N. Berkovits and C.R. Mafra, Equivalence of two-loop superstring amplitudes in the pure spinor and RNS formalisms, Phys. Rev. Lett. 96 (2006) 011602 [hep-th/0509234] [INSPIRE].

[4] C.R. Mafra, O. Schlotterer and S. Stieberger, Complete N-Point Superstring Disk Amplitude I. Pure Spinor Computation, Nucl. Phys. B 873 (2013) 419 [arXiv:1106.2645] [InSPIRE].

[5] H. Gomez and C.R. Mafra, The closed-string 3-loop amplitude and S-duality, JHEP 10 (2013) 217 [arXiv:1308.6567] [INSPIRE].

[6] H. Gomez, C.R. Mafra and O. Schlotterer, Two-loop superstring five-point amplitude and S-duality, Phys. Rev. D 93 (2016) 045030 [arXiv:1504.02759] [INSPIRE].

[7] C.R. Mafra, O. Schlotterer, S. Stieberger and D. Tsimpis, A recursive method for SYM n-point tree amplitudes, Phys. Rev. D 83 (2011) 126012 [arXiv:1012.3981] [INSPIRE].

[8] C.R. Mafra and O. Schlotterer, Towards one-loop SYM amplitudes from the pure spinor BRST cohomology, Fortsch. Phys. 63 (2015) 105 [arXiv:1410.0668] [InSPIRE].

[9] C.R. Mafra and O. Schlotterer, Two-loop five-point amplitudes of super Yang-Mills and supergravity in pure spinor superspace, JHEP 10 (2015) 124 [arXiv: 1505. 02746] [INSPIRE].

[10] N. Berkovits, Super Poincaré covariant quantization of the superstring, JHEP 04 (2000) 018 [hep-th/0001035] [INSPIRE].

[11] C.R. Mafra, Four-point one-loop amplitude computation in the pure spinor formalism, JHEP 01 (2006) 075 [hep-th/0512052] [INSPIRE].

[12] C.R. Mafra and O. Schlotterer, The Structure of n-Point One-Loop Open Superstring Amplitudes, JHEP 08 (2014) 099 [arXiv: 1203.6215] [INSPIRE].

[13] C.R. Mafra and O. Schlotterer, Multiparticle SYM equations of motion and pure spinor BRST blocks, JHEP 07 (2014) 153 [arXiv: 1404.4986] [inSPIRE].

[14] N. Berkovits and C.R. Mafra, Some Superstring Amplitude Computations with the Non-Minimal Pure Spinor Formalism, JHEP 11 (2006) 079 [hep-th/0607187] [INSPIRE].

[15] A. Tsuchiya, More on One Loop Massless Amplitudes of Superstring Theories, Phys. Rev. D 39 (1989) 1626 [inSPIRE].

[16] L. Clavelli, P.H. Cox and B. Harms, Parity Violating One Loop Six Point Function in Type I Superstring Theory, Phys. Rev. D 35 (1987) 1908 [inSPIRE].

[17] T. Inami, H. Kanno and T. Kubota, Hexagon Gauge Anomaly and Supermoduli in the Path Integral Method of Superstrings, Nucl. Phys. B 308 (1988) 203 [INSPIRE].

[18] N. Berkovits, Pure spinor formalism as an $N=2$ topological string, JHEP 10 (2005) 089 [hep-th/0509120] [INSPIRE].

[19] I. Oda and M. Tonin, Y-formalism in pure spinor quantization of superstrings, Nucl. Phys. B 727 (2005) 176 [hep-th/0505277] [INSPIRE].

[20] E. Witten, Twistor-like transform in ten-dimensions, Nucl. Phys. B 266 (1986) 245 [INSPIRE].

[21] M.B. Green and J.H. Schwarz, The Hexagon Gauge Anomaly in Type I Superstring Theory, Nucl. Phys. B 255 (1985) 93 [INSPIRE].

[22] M.B. Green and J.H. Schwarz, Anomaly Cancellation in Supersymmetric D = 10 Gauge Theory and Superstring Theory, Phys. Lett. B 149 (1984) 117 [inSPIRE]. 
[23] D. Friedan, E.J. Martinec and S.H. Shenker, Conformal Invariance, Supersymmetry and String Theory, Nucl. Phys. B 271 (1986) 93 [inSPIRE].

[24] C.R. Mafra and C. Stahn, The One-loop Open Superstring Massless Five-point Amplitude with the Non-Minimal Pure Spinor Formalism, JHEP 03 (2009) 126 [arXiv:0902.1539] [INSPIRE].

[25] S. Lee, C.R. Mafra and O. Schlotterer, Non-linear gauge transformations in D $=10 S Y M$ theory and the BCJ duality, JHEP 03 (2016) 090 [arXiv:1510.08843] [INSPIRE].

[26] C.R. Mafra and O. Schlotterer, Cohomology foundations of one-loop amplitudes in pure spinor superspace, arXiv:1408.3605 [INSPIRE].

[27] C.R. Mafra and O. Schlotterer, http://www.damtp.cam.ac.uk/user/crm66/SYM/pss.html.

[28] J. Broedel, C.R. Mafra, N. Matthes and O. Schlotterer, Elliptic multiple zeta values and one-loop superstring amplitudes, JHEP 07 (2015) 112 [arXiv:1412.5535] [INSPIRE].

[29] A. Weil, Elliptic Functions according to Eisenstein and Kronecker, Springer-Verlag, Heidelberg Germany (1976).

[30] D.M. Richards, The One-Loop Five-Graviton Amplitude and the Effective Action, JHEP 10 (2008) 042 [arXiv:0807.2421] [INSPIRE].

[31] J. Polchinski and Y. Cai, Consistency of Open Superstring Theories, Nucl. Phys. B 296 (1988) 91 [INSPIRE].

[32] L.A. Barreiro and R. Medina, RNS derivation of N-point disk amplitudes from the revisited S-matrix approach, Nucl. Phys. B 886 (2014) 870 [arXiv:1310.5942] [INSPIRE].

[33] L.A. Barreiro and R. Medina, Revisiting the S-matrix approach to the open superstring low energy effective lagrangian, JHEP 10 (2012) 108 [arXiv:1208.6066] [INSPIRE].

[34] S. Stieberger and T.R. Taylor, NonAbelian Born-Infeld action and type-I. Heterotic duality (1): Heterotic $F^{6}$ terms at two loops, Nucl. Phys. B 647 (2002) 49 [hep-th/0207026] [INSPIRE].

[35] S. Stieberger and T.R. Taylor, NonAbelian Born-Infeld action and type 1. - heterotic duality 2: Nonrenormalization theorems, Nucl. Phys. B 648 (2003) 3 [hep-th/0209064] [InSPIRE].

[36] S. He, R. Monteiro and O. Schlotterer, String-inspired BCJ numerators for one-loop MHV amplitudes, JHEP 01 (2016) 171 [arXiv:1507.06288] [INSPIRE].

[37] M.B. Green, C.R. Mafra and O. Schlotterer, Multiparticle one-loop amplitudes and S-duality in closed superstring theory, JHEP 10 (2013) 188 [arXiv:1307.3534] [INSPIRE].

[38] N.E.J. Bjerrum-Bohr and P. Vanhove, Explicit Cancellation of Triangles in One-loop Gravity Amplitudes, JHEP 04 (2008) 065 [arXiv: 0802.0868] [INSPIRE].

[39] M.B. Green and P. Vanhove, The Low-energy expansion of the one loop type-II superstring amplitude, Phys. Rev. D 61 (2000) 104011 [hep-th/9910056] [INSPIRE].

[40] M.B. Green, J.G. Russo and P. Vanhove, Low energy expansion of the four-particle genus-one amplitude in type-II superstring theory, JHEP 02 (2008) 020 [arXiv:0801.0322] [INSPIRE].

[41] M.B. Green, J.H. Schwarz and L. Brink, $N=4$ Yang-Mills and $N=8$ Supergravity as Limits of String Theories, Nucl. Phys. B 198 (1982) 474 [InSPIRE].

[42] M.B. Green and M. Gutperle, Effects of D instantons, Nucl. Phys. B 498 (1997) 195 [hep-th/9701093] [INSPIRE].

[43] M.B. Green and S. Sethi, Supersymmetry constraints on type IIB supergravity, Phys. Rev. D 59 (1999) 046006 [hep-th/9808061] [INSPIRE]. 
[44] A. Sinha, The $\hat{G}^{4} \lambda^{16}$ term in IIB supergravity, JHEP 08 (2002) 017 [hep-th/0207070] [INSPIRE].

[45] O. Schlotterer and S. Stieberger, Motivic Multiple Zeta Values and Superstring Amplitudes, J. Phys. A 46 (2013) 475401 [arXiv:1205.1516] [InSPIRE].

[46] N.E.J. Bjerrum-Bohr, P.H. Damgaard, T. Sondergaard and P. Vanhove, The Momentum Kernel of Gauge and Gravity Theories, JHEP 01 (2011) 001 [arXiv:1010.3933] [INSPIRE].

[47] H. Kawai, D.C. Lewellen and S.H.H. Tye, A Relation Between Tree Amplitudes of Closed and Open Strings, Nucl. Phys. B 269 (1986) 1 [InSPIRE].

[48] Z. Bern, L.J. Dixon, M. Perelstein and J.S. Rozowsky, Multileg one loop gravity amplitudes from gauge theory, Nucl. Phys. B 546 (1999) 423 [hep-th/9811140] [INSPIRE].

[49] J. Broedel, O. Schlotterer and S. Stieberger, http://mzv.mpp.mpg.de.

[50] D. Oprisa and S. Stieberger, Six gluon open superstring disk amplitude, multiple hypergeometric series and Euler-Zagier sums, hep-th/0509042 [INSPIRE].

[51] S. Stieberger and T.R. Taylor, Amplitude for N-Gluon Superstring Scattering, Phys. Rev. Lett. 97 (2006) 211601 [hep-th/0607184] [INSPIRE].

[52] J. Broedel, O. Schlotterer, S. Stieberger and T. Terasoma, All order $\alpha^{\prime}$-expansion of superstring trees from the Drinfeld associator, Phys. Rev. D 89 (2014) 066014 [arXiv: 1304.7304] [INSPIRE].

[53] J.M. Drummond and É. Ragoucy, Superstring amplitudes and the associator, JHEP 08 (2013) 135 [arXiv: 1301.0794] [INSPIRE].

[54] R.H. Boels, Maximal R-symmetry violating amplitudes in type IIB superstring theory, Phys. Rev. Lett. 109 (2012) 081602 [arXiv:1204.4208] [INSPIRE].

[55] R.H. Boels, On the field theory expansion of superstring five point amplitudes, Nucl. Phys. B 876 (2013) 215 [arXiv: 1304.7918] [INSPIRE].

[56] M. Hayashi, N. Kawamoto, T. Kuramoto and K. Shigemoto, Modular Invariance and Gravitational Anomaly in Type II Superstring Theory, Nucl. Phys. B 294 (1987) 459 [INSPIRE].

[57] D. Kutasov, Modular Invariance, Chiral Anomalies and Contact Terms, Nucl. Phys. B 307 (1988) 417 [inSPIRE].

[58] W. Lerche, A.N. Schellekens and N.P. Warner, Lattices and Strings, Phys. Rept. 177 (1989) 1 [INSPIRE].

[59] M. Berg, I. Buchberger and O. Schlotterer, From maximal to minimal supersymmetry in string loop amplitudes, arXiv:1603.05262 [INSPIRE].

[60] N. Berkovits and E. Witten, Supersymmetry Breaking Effects using the Pure Spinor Formalism of the Superstring, JHEP 06 (2014) 127 [arXiv: 1404.5346] [INSPIRE].

[61] D.J. Gross and P.F. Mende, Modular Subgroups, Odd Spin Structures and Gauge Invariance in the Heterotic String, Nucl. Phys. B 291 (1987) 653 [INSPIRE].

[62] C.R. Mafra and O. Schlotterer, Solution to the nonlinear field equations of ten dimensional supersymmetric Yang-Mills theory, Phys. Rev. D 92 (2015) 066001 [arXiv:1501.05562] [INSPIRE]. 